\title{
Verification and Strengthening of the Atiyah-Sutcliffe Conjectures for Several Types of Almost Collinear Configurations in Euclidean and Hyperbolic Plane
}

\author{
Dragutin Svrtan \\ Department of Mathematics, University of Zagreb, \\ Bijenička cesta 30, 10000 Zagreb, Croatia, \\ dsvrtan@math.hr \\ Igor Urbiha \\ Department of Informatics, Polytechnic of Zagreb, University of Zagreb, \\ Konavoska 2, 10000 Zagreb, Croatia, \\ igor.urbiha@tvz.hr
}

\begin{abstract}
In 2001 Sir M. F. Atiyah formulated a conjecture C1 and later with P. Sutcliffe two stronger conjectures $\mathrm{C} 2$ and $\mathrm{C} 3$. These conjectures, inspired by physics (spin-statistics theorem of quantum mechanics), are geometrically defined for any configuration of points in the Euclidean three space. The conjecture $\mathrm{C} 1$ is proved for $n=3,4$ and for general $n$ only for some special configurations (M. F. Atiyah, M. Eastwood and P. Norbury, D.Đoković). In [11] and [12] we have verified the conjectures $\mathrm{C} 2$ and $\mathrm{C} 3$ for parallelograms, cyclic quadrilaterals and some infinite families of tetrahedra, and have proposed a strengthening of conjecture C3 for configurations of four points (Four Points Conjectures). All three Euclidean four-point conjectures have been proved in [14] (2010.) (see also [15] for more information) then independently four years later in [10].

For almost collinear configurations of type A (with all but one point on a line) we propose in [12] several new conjectures (some for symmetric functions) which imply C2 and C3. By using computations with multi-Schur functions we can do verifications up to $n=9$ of our conjectures. For a type B (and some special dihedral configurations) we verify a stronger conjecture of Đoković which implies C2 for his nonplanar configurations with dihedral symmetry. Recently
\end{abstract}

DOI: https://doi.org/10.5592/CO/CCD.2018.10 
we have observed that our generalizations from [12] are capable to imply hyperbolic $\mathrm{C} 2$ for type $\mathrm{A}$ and $\mathrm{B}$ configurations and we make these connections clear in Sections 1.2 and 1.3.

Finally we mention that by minimizing a geometrically defined energy, figuring in these conjectures, one gets a connection to some complicated physical theories, such as Skyrmions and Fullerenes.

Keywords: Atiyahs conjecture, Configuration of $\mathrm{N}$ points in the three-dimensional Euclidean space, Configuration of N points in the Hyperbolic plane

MSC: 51M04, 51M16, 70G25

\section{Almost collinear configurations. Đoković's approach}

\subsection{Type A configurations}

By a type A configurations of $N$ points $x_{1}, \ldots, x_{N}$ we shall mean the case when $N-1$ of the points $x_{1}, \ldots, x_{N}$ are collinear. Set $n=N-1$. In ([6]) Đoković has proved, for configurations of type A, both the Atiyah conjecture (Theorem 2.1) and the first Atiyah-Sutcliffe conjecture (Theorem 3.1). By using Cartesian coordinates, with $x_{i}=\left(a_{i}, 0\right), a_{1}<a_{2}<\cdots<a_{n}$ and $x_{N}=x_{n+1}=(0, b)$ (with $\left.b=1\right)$, the normalized Atiyah matrix $M_{n+1}=M_{n+1}\left(\lambda_{1}, \ldots, \lambda_{n}\right)$ (denoted by $P$ in [6] when $b=-1)$ is given by

$$
M_{n+1}=\left[\begin{array}{cccccc}
1 & \lambda_{1} & 0 & \cdots & 0 & 0 \\
0 & 1 & \lambda_{2} & \cdots & 0 & 0 \\
0 & 0 & 1 & & 0 & 0 \\
\vdots & \vdots & \vdots & \ddots & \vdots & \vdots \\
0 & 0 & & & 1 & \lambda_{n} \\
(-1)^{n} e_{n} & (-1)^{n-1} e_{n-1} & \cdots & \cdots & -e_{1} & 1
\end{array}\right]
$$

where $\lambda_{1}=a_{1}+\sqrt{a_{1}^{2}+b^{2}}<\lambda_{2}=a_{2}+\sqrt{a_{2}^{2}+b^{2}}<\cdots<\lambda_{n}=a_{n}+\sqrt{a_{n}^{2}+b^{2}}$ (with $b=1$ ) are positive real numbers and where $e_{k}=e_{k}\left(\lambda_{1}, \ldots, \lambda_{n}\right), 1 \leq k \leq n$, is the $k$-th elementary symmetric function of $\lambda_{1}, \lambda_{2}, \ldots, \lambda_{n}$. Its determinant satisfies the inequality

$$
\begin{aligned}
D_{n} & =1+\lambda_{n} e_{1}+\lambda_{n} \lambda_{n-1} e_{2}+\cdots+\lambda_{n} \lambda_{n-1} \cdots \lambda_{1} e_{n} \\
& \geq 1+e_{1}\left(\lambda_{1}^{2}, \ldots, \lambda_{n}^{2}\right)+e_{2}\left(\lambda_{1}^{2}, \ldots, \lambda_{n}^{2}\right)+\cdots+e_{n}\left(\lambda_{1}^{2}, \ldots, \lambda_{n}^{2}\right) \\
& =\prod_{i=1}^{n}\left(1+\lambda_{i}^{2}\right)
\end{aligned}
$$


equivalent to the first Atiyah-Sutcliffe conjecture ([4],Conjecture 2). The second Atiyah-Sutcliffe conjecture ([4], Conjecture 3) for configurations of type A is equivalent to the following inequality

$$
\left[D_{n+1}\left(\lambda_{1}, \ldots, \lambda_{n}\right)\right]^{n-1} \geq \prod_{k=1}^{n} D_{n}\left(\lambda_{1}, \ldots, \lambda_{k-1}, \lambda_{k+1}, \ldots, \lambda_{n}\right)
$$

For $n=2$ this inequality takes the form

$$
1+\lambda_{2} e_{1}\left(\lambda_{1}, \lambda_{2}\right)+\lambda_{1} \lambda_{2} e_{2}\left(\lambda_{1}, \lambda_{2}\right) \geq\left(1+\lambda_{2} e_{1}\left(\lambda_{2}\right)\right)\left(1+\lambda_{1} e_{1}\left(\lambda_{1}\right)\right.
$$

i.e.

$$
1+\lambda_{2} e_{1}\left(\lambda_{1}, \lambda_{2}\right)+\lambda_{1} \lambda_{2} e_{2}\left(\lambda_{1}, \lambda_{2}\right) \geq\left(1+\lambda_{2}^{2}\right)\left(1+\lambda_{1}^{2}\right) .
$$

This reduces to $\left(\lambda_{2}-\lambda_{1}\right) \lambda_{1} \geq 0$, so it is true.

\subsection{Type A configurations - hyperbolic case}

Let $H^{2}=\left\{(x, y) \in \mathbb{R}^{2} \mid y>0\right\}$ be the upper half plane model of hyperbolic plane. A type A hyperbolic configuration denoted by $A_{n, 1}=A\left(\mathbf{x}_{1}, \mathbf{x}_{2}, \ldots, \mathbf{x}_{n} ; \mathbf{x}_{n+1}\right)$ consists of $N=n+1$ points in $H^{2}$ where $n$ points are collinear, $\left(\mathbf{x}_{1}, \mathbf{x}_{2}, \ldots, \mathbf{x}_{n}\right), \mathbf{x}_{i}=\left(0, b_{i}\right)$, $b_{1}>b_{2}>\cdots>b_{n}>0$ and $\mathbf{x}_{n+1}=(a, b)$.

Let us abbreviate the directions $t_{i j}$ from $\mathbf{x}_{i}$ to $\mathbf{x}_{j}$ (viewed as points on the absolute $\mathbb{R} \cup\{\infty\})$

$$
\begin{array}{ll}
t_{i, n+1}=X_{i}, 1 \leq i \leq n & t_{i j}=0 \text { for } 1 \leq i<j \leq n \\
t_{n+1, i}=-\xi_{i}, 1 \leq i \leq n & t_{j i}=\infty \text { for } 1 \leq i<j \leq n .
\end{array}
$$

Then we have $t_{i, n+1} \cdot t_{n+1, i}=-b_{i}^{2}$ i.e. $\xi_{i} X_{i}=b_{i}^{2}$.

The Atiyah polynomials associated to points $\mathbf{x}_{i}(1 \leq i \leq n+1)$ defined by

$$
p_{i}=\prod_{j \neq i}\left(z-t_{i j}\right)
$$

(with $z-t_{i j}$ interpreted as 1 if $t_{i j}=\infty$ ) are given explicitly as follows

$$
\begin{aligned}
& p_{1}=z^{n-1}\left(z-X_{1}\right) \\
& p_{2}=z^{n-2}\left(z-X_{2}\right) \\
& \quad \quad \vdots \\
& p_{n-1}=z\left(z-X_{n-1}\right) \\
& p_{n}=z-X_{n} \\
& p_{n+1}=\left(z+\xi_{1}\right)\left(z+\xi_{2}\right) \cdots\left(z+\xi_{n}\right)=z^{n}+e_{1} z^{n-1}+\cdots+e_{n},
\end{aligned}
$$


where $e_{1}, \ldots, e_{n}$ denotes the elementary symmetric functions of $\xi_{1}, \ldots, \xi_{n}$. The Atiyah determinant (the determinant of the matrix of coefficients of $p_{i}$ 's) $D_{n, 1}^{\text {hyp }}=$ $D_{n, 1}^{\text {hyp }}\left(\mathbf{x}_{1}, \mathbf{x}_{2}, \ldots, \mathbf{x}_{n} ; \mathbf{x}_{n+1}\right)$ is then equal to

$$
D_{n, 1}^{\text {hyp }}=\left|\begin{array}{cccccc}
1 & -X_{1} & & & & \\
0 & 1 & -X_{2} & & & \\
\vdots & \vdots & \vdots & \ddots & & \\
& & & & 1 & -X_{n} \\
1 & e_{1} & e_{2} & \ldots & e_{n-1} & e_{n}
\end{array}\right|=\sum_{i=0}^{n} e_{i} \cdots X_{n}+X_{2} \cdots X_{n} e_{1}+\cdots+e_{n}=
$$




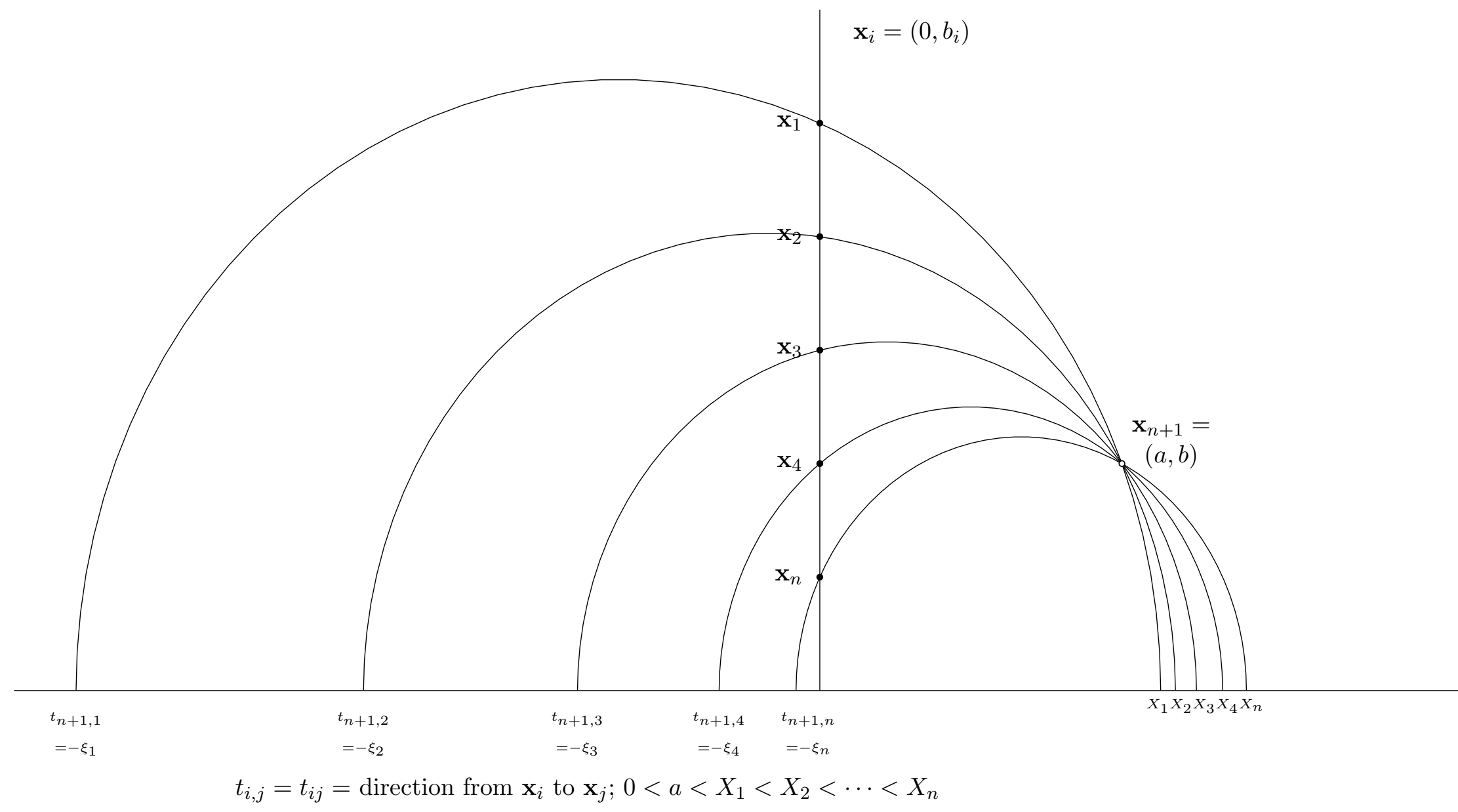


Note that

$$
\left.X_{1} \cdots X_{n} \cdot D_{n, 1}^{\text {hyp }}\right|_{X_{i} \rightarrow X_{i}^{-1}}
$$

is a specialization of the polynomial

$$
\Psi_{12 \ldots n}^{12 \ldots n} \in Q\left[X_{1}, \ldots, X_{n}, \xi_{1}, \ldots, \xi_{n}\right]
$$

defined in the section 1.4 which is symmetric in $\xi_{1}, \ldots, \xi_{n}$. The properties of polynomials $\Psi_{J}^{I}$ will enable us to study simultaneously both the euclidean and the hyperbolic configurations of type A (and B, defined later). Since

$$
\left|\begin{array}{ll}
1 & -t_{j, n+1} \\
1 & -t_{n+1, j}
\end{array}\right|=t_{j, n+1}-t_{n+1, j}=X_{j}+\xi_{j}, 1 \leq j \leq n
$$

we obtain for the normalized Atiyah determinant the following formula

$$
D_{n, 1}=\frac{\sum_{i=0}^{n} e_{i} X_{i+1} \cdots X_{n}}{\prod_{j=1}^{n}\left(X_{j}+\xi_{j}\right)} \quad\left(\text { with } \xi_{i} X_{i}=b_{i}^{2}\right) .
$$

Lemma 1.1 For $0<X_{1} \leq X_{2} \leq \ldots \leq X_{n}$ and $\xi_{1}, \ldots, \xi_{n}$ commuting indeterminates, the inequality

$$
\prod_{j=1}^{n}\left(X_{j}+\xi_{j}\right) \leq \sum_{i=0}^{n} e_{i}\left(\xi_{1}, \ldots, \xi_{n}\right) X_{i+1} \cdots X_{n}
$$

holds true coefficientwise.

Proof .

$$
\begin{aligned}
\prod_{j=1}^{n}\left(X_{j}+\xi_{j}\right)=\sum_{S \subseteq[n]} \prod_{j \in S} \xi_{j} \cdot \prod_{k \in S^{C}} X_{k} & \leq \sum_{S \subseteq[n]} \prod_{j \in S} \xi_{j} \cdot X_{|S|+1} \cdots X_{n} \\
& =\sum_{i=0}^{n} e_{i} \cdot X_{i+1} \cdots X_{n}
\end{aligned}
$$


Corollary 1.2 For any $n$ points on a line and one point outside it in a hyperbolic plane (or space) we have that the second Atiyah-Sutcliffe conjecture holds true:

$$
D_{n, 1}^{h y p}\left(\mathbf{x}_{1}, \ldots, \mathbf{x}_{n}, \mathbf{x}_{n+1}\right) \geq \prod_{1 \leq i<j \leq n+1} D_{2}\left(\mathbf{x}_{i}, \mathbf{x}_{j}\right)=\prod_{j=1}^{n} D_{2}\left(\mathbf{x}_{j}, \mathbf{x}_{n+1}\right) .
$$

Proof .

We have that 1.h.s. is equal to $\sum e_{i} X_{i+1} \cdots X_{n}$ and the r.h.s. reduces to the product $\prod_{j=1}^{n}\left(X_{j}+\xi_{j}\right)$.

\subsection{Type B configurations - hyperbolic case}

Let $\mathbf{x}_{i}=\left(0, b_{i}\right), b_{1}>b_{2}>\cdots>b_{r}>c>b_{r+1}>\cdots>b_{n}>0,\left(c=\sqrt{a^{2}+b^{2}}\right)$, $\mathbf{x}_{n+1}=(a, b)$ and $\mathbf{x}_{n+2}=(-a, b)$ be a type B hyperbolic configuration of $N=n+2$ points in $H^{2}$ denoted by $B_{n, 2}=B\left(\mathbf{x}_{1}, \mathbf{x}_{2}, \ldots, \mathbf{x}_{n} ; \mathbf{x}_{n+1}, \mathbf{x}_{n+2}\right)$.

The hyperbolic directions from $x_{i}$ to $x_{j}$ we abbreviate as follows

$$
\begin{array}{ll}
t_{i, n+1}=X_{i}, 1 \leq i \leq n & t_{i j}=0 \text { for } 1 \leq i<j \leq n \\
t_{n+1, i}=-\xi_{i}, 1 \leq i \leq n & t_{j i}=\infty \text { for } 1 \leq i<j \leq n \\
t_{i, n+2}=-X_{i}, 1 \leq i \leq n & t_{n+1, n+2}=-c \\
t_{n+2, i}=\xi_{i}, 1 \leq i \leq n & t_{n+2, n+1}=c .
\end{array}
$$

The Atiyah polynomials associated to points $\mathbf{x}_{i}(1 \leq i \leq n+2)$ defined (in [1] - [4]) by

$$
p_{i}=\prod_{j \neq i}\left(z-t_{i j}\right)
$$

(with $z-t_{i j}$ interpreted as 1 if $t_{i j}=\infty$ ) associated to points $\mathbf{x}_{i}(1 \leq i \leq n+2)$ are then

$$
\begin{aligned}
& p_{1}=z^{n-1}\left(z-X_{1}\right)\left(z+X_{1}\right) \\
& p_{2}=z^{n-2}\left(z-X_{2}\right)\left(z+X_{2}\right) \\
& \quad \quad \vdots \\
& p_{n-1}=z\left(z-X_{n-1}\right)\left(z+X_{n-1}\right) \\
& p_{n}=\left(z-X_{n}\right)\left(z+X_{n}\right) \\
& p_{n+1}=\left(z+\xi_{1}\right)\left(z+\xi_{2}\right) \cdots\left(z+\xi_{n}\right)(z+c)=z^{n+1}+e_{1} z^{n}+\cdots+e_{n+1} \\
& p_{n+2}=\left(z-\xi_{1}\right)\left(z-\xi_{2}\right) \cdots\left(z-\xi_{n}\right)(z-c)=z^{n+1}-e_{1} z^{n}+\cdots+(-1)^{n+1} e_{n+1} .
\end{aligned}
$$




$$
(-1)^{n+1} D_{n, 2}^{\text {hyp }}=(-1)^{n+1}\left|\begin{array}{ccccccc}
1 & 0 & -X_{1}^{2} & 0 & & 0 \\
0 & 1 & 0 & -X_{2}^{2} & & 0 \\
\vdots & & & & \ddots & & \\
0 & 0 & 0 & & 1 & 0 & -X_{n}^{2} \\
1 & e_{1} & e_{2} & e_{3} & \cdots & e_{n} & e_{n+1} \\
1 & -e_{1} & e_{2} & -e_{3} & \cdots & & (-1)^{n+1} e_{n+1}
\end{array}\right|=
$$

We first add $n+1$-st row to the $n+2$-nd row and then subtract a half of the $n+2$-nd row from the $n+1$-st row.

$$
=(-1)^{n+1} 2\left|\begin{array}{ccccccc}
1 & 0 & -X_{1}^{2} & 0 & & & 0 \\
0 & 1 & 0 & -X_{2}^{2} & & & 0 \\
\vdots & & & & \ddots & & \\
0 & 0 & 0 & & 1 & 0 & -X_{n}^{2} \\
0 & e_{1} & 0 & e_{3} & \cdots & 0 & e_{n+1} \\
1 & 0 & e_{2} & 0 & \cdots & e_{n} & 0
\end{array}\right|(n \text { even })=\ldots
$$

Then we add $-1 \cdot 1^{\text {st }}$ row $-\left(X_{1}^{2}+e_{2}\right) \cdot 3^{\text {rd }}$ row $+X_{3}^{2}\left(X_{1}^{2}+e_{2}\right) \cdot 5^{\text {th }}$ row $+\cdots$ to the $n+2$-nd row and we also add $-e_{1} \cdot 2^{\text {nd }}$ row $+\left(-\left(X_{2}^{2} e_{1}+e_{3}\right)\right) \cdot 4^{\text {th }}$ row $+\cdots$ to the $n+1$-st row.

$$
=2\left|\begin{array}{cccccccc}
1 & 0 & -X_{1}^{2} & 0 & 0 & & & 0 \\
0 & 1 & 0 & -X_{2}^{2} & 0 & & & 0 \\
0 & 0 & 1 & 0 & -X_{3}^{2} & & & 0 \\
0 & 0 & 0 & 1 & 0 & -X_{4}^{2} & & 0 \\
0 & 0 & 0 & 0 & 1 & 0 & -X_{5}^{2} & 0 \\
\vdots & & & & \vdots & & & \vdots \\
0 & 0 & 0 & X_{2}^{2} e_{1}+e_{3} & 0 & e_{5} & \ldots & \\
0 & 0 & X_{1}^{2}+e_{2} & 0 & e_{4} & \ldots & &
\end{array}\right|=\ldots
$$

At the end we get a triangular matrix whose determinant is equal to $D_{n, 2}^{\text {hyp }}=2 f_{0} f_{1}$ where we have for $n$ even

$f_{n, 2}^{0}=X_{1}^{2} X_{3}^{2} \cdots X_{n-1}^{2}+X_{3}^{2} \cdots X_{n-1}^{2} e_{2}+\cdots+X_{n-1}^{2} e_{n-2}+e_{n}$ $f_{n, 2}^{1}=X_{2}^{2} X_{4}^{2} \cdots X_{n}^{2} e_{1}+X_{4}^{2} \cdots X_{n}^{2} e_{3}+\cdots+X_{n}^{2} e_{n-1}+e_{n+1}$,

and for $n$ odd

$f_{n, 2}^{0}=X_{1}^{2} X_{3}^{2} \cdots X_{n}^{2}+X_{3}^{2} \cdots X_{n}^{2} e_{2}+\cdots+X_{n}^{2} e_{n-1}+e_{n+1}$

$f_{n, 2}^{1}=X_{2}^{2} X_{4}^{2} \cdots X_{n-1}^{2} e_{1}+X_{4}^{2} \cdots X_{n-1}^{2} e_{3}+\cdots+X_{n-1}^{2} e_{n-2}+e_{n}$. 
For example

$$
\begin{aligned}
& D_{1,2}^{\text {hyp }}=2\left(X_{1}^{2}+e_{2}\right) e_{1} \\
& D_{2,2}^{\text {hyp }}=2\left(X_{1}^{2}+e_{2}\right)\left(X_{2}^{2} e_{1}+e_{3}\right) \\
& D_{3,2}^{\text {hyp }}=2\left(X_{1}^{2} X_{3}^{2}+X_{3}^{2} e_{2}+e_{4}\right)\left(X_{2}^{2} e_{1}+e_{3}\right) \\
& D_{4,2}^{\text {hyp }}=2\left(X_{1}^{2} X_{3}^{2}+X_{3}^{2} e_{2}+e_{4}\right)\left(X_{2}^{2} X_{4}^{2} e_{1}+X_{4}^{2} e_{3}+e_{5}\right)
\end{aligned}
$$

Now we shall verify Atiyah-Sutcliffe Conjecture 2 for the hyperbolic configuration $\mathrm{B}_{n, 2}$ of the type $\mathrm{B}$ which reads as follows

$$
D_{n, 2}^{\text {hyp }} \geq\left(X_{1}+\xi_{1}\right)^{2}\left(X_{2}+\xi_{2}\right)^{2} \cdots\left(X_{n}+\xi_{n}\right)^{2} 2 c
$$

Case 1. ( $n$ even) We first rewrite $f_{n, 2}^{0}$ and $f_{n, 2}^{1}$ in terms of $c$ and elementary symmetric functions $e_{k}^{\prime}(1 \leq k \leq n)$ of $\xi_{1}, \ldots \xi_{n}$ :

$$
\begin{aligned}
f_{n, 2}^{0}=X_{1}^{2} X_{3}^{2} \cdots X_{n-1}^{2} & +X_{3}^{2} \cdots X_{n-1}^{2} e_{2}^{\prime}+\cdots+X_{n-1}^{2} e_{n-2}^{\prime}+e_{n}^{\prime} \\
& +X_{3}^{2} \cdots X_{n-1}^{2} e_{1}^{\prime} c+\cdots+X_{n-1}^{2} e_{n-3}^{\prime} c+e_{n-1}^{\prime} c \\
f_{n, 2}^{1}= & X_{2}^{2} X_{4}^{2} \cdots X_{n}^{2} e_{1}^{\prime}+X_{4}^{2} \cdots X_{n}^{2} e_{3}^{\prime}+\cdots+X_{n}^{2} e_{n-1}^{\prime}+e_{n+1}^{\prime} \\
& X_{2}^{2} X_{4}^{2} \cdots X_{n}^{2} c+X_{4}^{2} \cdots X_{n}^{2} e_{2}^{\prime} c+\cdots+X_{n}^{2} e_{n-2} c+e_{n}^{\prime} c
\end{aligned}
$$

Now by Cauchy-Schwartz

$$
\begin{aligned}
& D_{n, 2}^{\text {hyp }}=2 f_{n, 2}^{0} f_{n, 2}^{1} \geq \\
& \geq 2\left(X_{1} X_{3} \cdots X_{n-1} \cdot X_{2} X_{4} \cdots X_{n} \sqrt{c}+X_{3} X_{5} \cdots X_{n-1} \cdot X_{2} X_{4} \cdots X_{n} e_{1}^{\prime} \sqrt{c}+\cdots+e_{n}^{\prime} \sqrt{c}\right)^{2}= \\
& =\left(X_{1} X_{2} \cdots X_{n}+X_{2} X_{3} \cdots X_{n} e_{1}^{\prime}+\cdots+X_{n} e_{n-1}^{\prime}+e_{n}^{\prime}\right)^{2} \cdot 2 c= \\
& =\left(D_{n, 1}^{\text {hyp }}\right)^{2} \cdot 2 c
\end{aligned}
$$

Now by Corollary 1.2 for $D_{n, 1}^{\text {hyp }}$ the inequality $(\star)$ follows.

Case 2. ( $n$ odd) Is similar to Case 1.

This concludes the verification of the Atiyah-Sutcliffe Conjecture C2 for hyperbolic type B configurations.

Now we state the strongest Atiyah-Sutcliffe conjecture C3 for type A and type B hyperbolic configurations.

\section{Conjecture 3.}


(i) $D_{n, 1}^{\text {hyp }}\left(\mathbf{x}_{1}, \ldots, \mathbf{x}_{n} ; \mathbf{x}_{n+1}\right)^{n-1} \geq \prod_{k=1}^{n} D_{n-1,1}^{\text {hyp }}\left(\mathbf{x}_{1}, \ldots \widehat{\mathbf{x}}_{k}, \ldots, \mathbf{x}_{n} ; \mathbf{x}_{n+1}\right)$ (a hyperbolic analogue of formula (1.1))

(ii) $D_{n, 2}^{\text {hyp }}\left(\mathbf{x}_{1}, \ldots, \mathbf{x}_{n} ; \mathbf{x}_{n+1}, \mathbf{x}_{n+2}\right)^{n} \geq D_{n, 1}^{\text {hyp }}\left(\mathbf{x}_{1}, \ldots, \mathbf{x}_{n} ; \mathbf{x}_{n+1}\right)$. $\cdot D_{n, 1}^{\text {hyp }}\left(\mathbf{x}_{1}, \ldots, \mathbf{x}_{n} ; \mathbf{x}_{n+2}\right) \prod_{k=1}^{n} D_{n-1,2}^{\text {hyp }}\left(\mathbf{x}_{1}, \ldots \widehat{\mathbf{x}}_{k}, \ldots, \mathbf{x}_{n} ; \mathbf{x}_{n+1}, \mathbf{x}_{n+2}\right)$

For example for $n=2$ we have

$$
\begin{aligned}
& \left(D_{2,2}^{\text {hyp }}\right)^{2}=4\left[X_{1}^{2}+\xi_{1} \xi_{2}+\left(\xi_{1}+\xi_{2}\right) c\right]^{2}\left[X_{2}^{2}\left(\xi_{1}+\xi_{2}\right)+\left(X_{2}^{2}+\xi_{1} \xi_{2}\right) c\right]^{2} \geq \\
& \geq\left(X_{1} X_{2}+X_{2}\left(\xi_{1}+\xi_{2}\right)+\xi_{1} \xi_{2}\right)^{2} \cdot\left(X_{1}^{2}+\xi_{1} c\right)\left(c+\xi_{2}\right) \cdot\left(X_{2}^{2}+\xi_{2} c\right)\left(c+\xi_{1}\right)
\end{aligned}
$$

which can be proved by considering the following three cases:

Case 1: $0<X_{1}<X_{2}<c<\xi_{2}<\xi_{1}$

Case 2: $0<\xi_{2}<X_{1}<c<X_{2}<\xi_{1}$

Case 3: $0<\xi_{2}<\xi_{1}<c<X_{1}<X_{2}$

and we get coefficient-wise inequality in terms of variables representing increments e.g. in Case 1: $h_{1}=X_{2}-X_{1}, h_{2}=c-X_{2}, h_{3}=\xi_{2}-c, h_{4}=\xi_{1}-\xi_{2}$ and $X_{1}$.

We expect that the general proof will not be so elementary.

For hyperbolic planar four points case the conjectures $\mathrm{C} 1$ and $\mathrm{C} 2$ were verified and reported in [16] and the conjecture $\mathrm{C} 1$ for non planar case of four points in $H^{3}$ were treated in [9] therefore conjecture $\mathrm{C} 1$ was verified for any four points in $H^{3}$. 


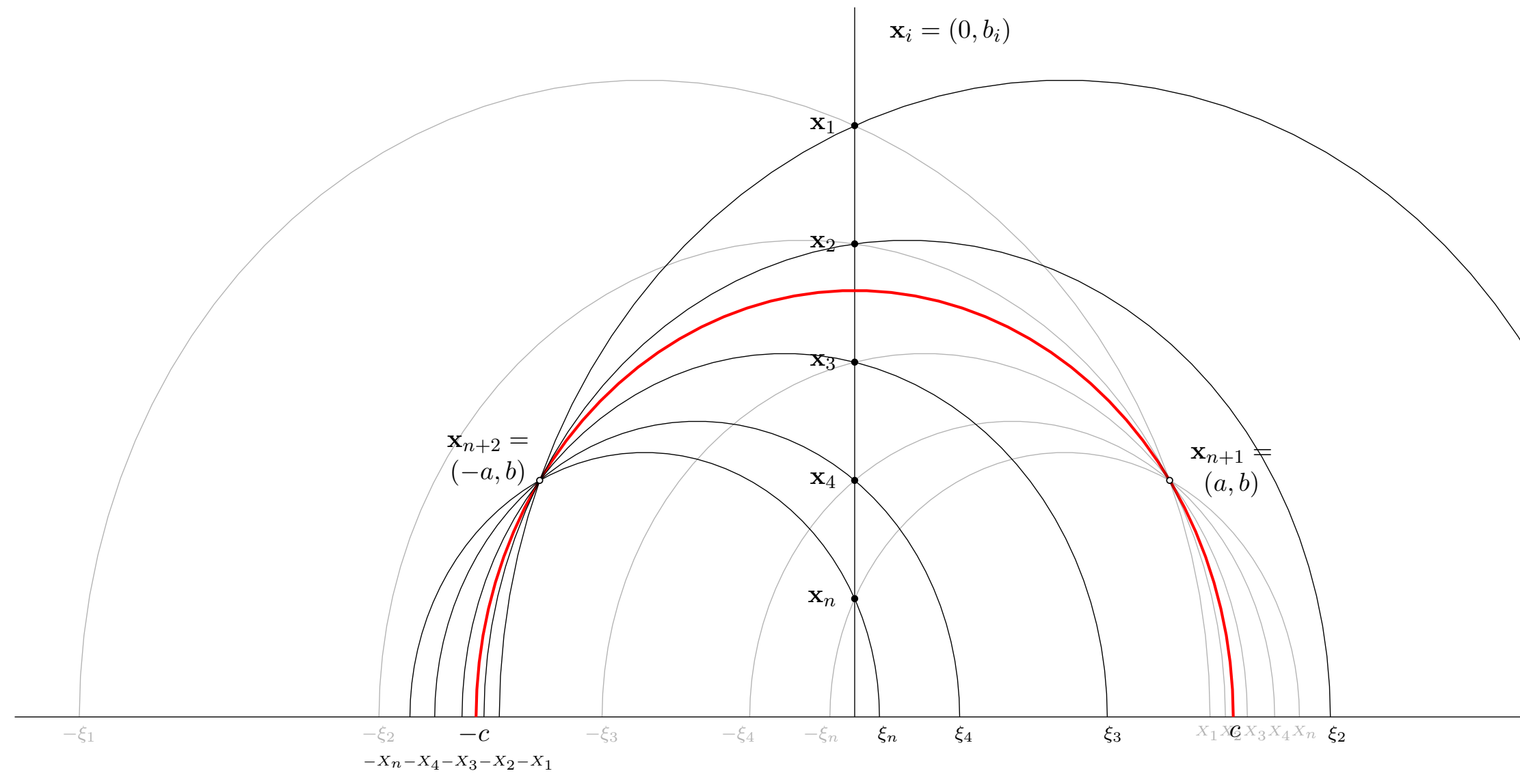

$t_{i, j}=t_{i j}=$ direction from $\mathbf{x}_{i}$ to $\mathbf{x}_{j} ; 0<a<X_{1}<X_{2}<\cdots<X_{n}$ 


\subsection{Type (A) configurations (cont.)}

Even for $n=3$ the inequality (1.1) is quite messy thanks to nonsymmetric character of both sides.

Knowing that sometimes it is easier to solve a more general problem we followed that path (although we didn't solve the problem in full generality). So let us start with the case $n=2$. If we look at the following inequality

$$
1+X_{1}\left(\xi_{1}+\xi_{2}\right)+X_{1} X_{2} \xi_{1} \xi_{2} \geq\left(1+X_{1} \xi_{1}\right)\left(1+X_{2} \xi_{2}\right)
$$

which is clearly true if $X_{1} \geq X_{2} \geq 0$ and $\xi_{1}, \xi_{2} \geq 0$ we obtain the inequality (1.2) simply by a specialization $X_{1}=\xi_{1}=\lambda_{2}, X_{2}=\xi_{2}=\lambda_{1}$. So we proceed as follows:

Let $\xi_{1}, \ldots, \xi_{n}, X_{1}, \ldots, X_{n}, n \geq 1$ be two sets of commuting indeterminates. For any $l, 1 \leq l \leq n$ and any sequences $1 \leq i_{1} \leq \cdots \leq i_{l} \leq n, 1 \leq j_{1}, \ldots, j_{l} \leq n$ we define polynomials $\Psi_{J}^{I}=\Psi_{j_{1} \ldots j_{l}}^{i_{1} \ldots i_{l}} \in \mathbb{Q}\left[\xi_{1}, \ldots, \xi_{n}, X_{1}, \ldots, X_{n}\right]$ as follows:

$$
\Psi_{J}^{I}:=\sum_{k=0}^{l} e_{k}\left(\xi_{j_{1}}, \xi_{j_{2}}, \ldots, \xi_{j_{l}}\right) X_{i_{1}} X_{i_{2}} \cdots X_{i_{k}}, \quad(l \geq 1), \Psi_{\emptyset}^{\emptyset}:=1(j=0)
$$

where $e_{k}$ is the $k$-th elementary symmetric function.

In particular we have

$$
\begin{aligned}
\Psi_{j}^{i}=1 & +\xi_{j} X_{i} \\
\Psi_{j_{1} j_{2}}^{i_{1} i_{2}}=1 & +\left(\xi_{j_{1}}+\xi_{j_{2}}\right) X_{i_{1}}+\xi_{j_{1}} \xi_{j_{2}} X_{i_{1}} X_{i_{2}} \\
\Psi_{j_{1} j_{2} j_{3}}^{i_{i} i_{3}}=1 & +\left(\xi_{j_{1}}+\xi_{j_{2}}+\xi_{j_{3}}\right) X_{i_{1}}+\left(\xi_{j_{1}} \xi_{j_{2}}+\xi_{j_{1}} \xi_{j_{3}}+\xi_{j_{2}} \xi_{j_{3}}\right) X_{i_{1}} X_{i_{2}}+ \\
& +\xi_{j_{1}} \xi_{j_{2}} \xi_{j_{3}} X_{i_{1}} X_{i_{2}} X_{i_{3}}
\end{aligned}
$$

etc.

The polynomials $\Psi_{J}^{I}$ are symmetric w.r.t. $\xi_{j_{1}}, \xi_{j_{2}}, \ldots, \xi_{j_{l}}$, but nonsymmetric w.r.t. $X_{i_{1}}, X_{i_{2}}, \ldots, X_{i_{l}}$. By specializing $X_{i}$ 's to assume real values such that $X_{i_{1}} \geq X_{i_{2}} \geq$ $\ldots \geq X_{i_{l}} \geq 0$ then we obtain polynomials in $\xi_{j}$ 's satisfying the following simple but important property.

Proposition 1.3 (Partition property)

Let $\left(I_{1}, \ldots, I_{s}\right)$ and $\left(J_{1}, \ldots, J_{s}\right)$ be ordered set partitions of respective sets $I=$ $\bigcup_{p=1}^{s} I_{p}$ and $J=\bigcup_{p=1}^{s} J_{p}$ such that $\left|I_{p}\right|=\left|J_{p}\right|, 1 \leq p \leq s$. Then the inequality

$$
\Psi_{J}^{I} \geq \prod_{p=1}^{s} \Psi_{J_{p}}^{I_{p}}
$$


holds coefficientwise w.r.t. $\xi_{j}$ 's.

Proof .

Proof is evident from the definition of $\Psi_{J}^{I}$ and the monotonicity of $X_{i}$ 's.

For the powers $\left(\Psi_{J}^{I}\right)^{m}$ we have the following conjecture.

Conjecture 1.4 (Weighted Multiset Partition Conjecture)

For given natural number $m$ and sets $I$ and $J,|I|=|J|$, of natural numbers let $\left(I_{1}, \ldots, I_{s}\right)$ and $\left(J_{1}, \ldots, J_{s}\right)$ be the partitions of the multiset $I^{m}$ consisting of $m$ copies of all elements of $I$ and similarly for $J^{m}$.

(i) Then the inequality

$$
\left(\Psi_{J}^{I}\right)^{m} \geq \prod_{p=1}^{s} \Psi_{J_{p}}^{I_{p}}
$$

holds coefficientwise w.r.t. $\xi_{j}$ 's.

(ii) The difference

$$
\left(\Psi_{J}^{I}\right)^{m}-\prod_{p=1}^{s} \Psi_{J_{p}}^{I_{p}}
$$

is multi-Schur positive with respect to partial alphabets corresponding to the atoms of the intersection lattice of the set system $\left\{J_{1}, \ldots, J_{s}\right\}$.

For example, by Partition property, we have the following inequalities

$$
\Psi_{1 \ldots n}^{1 \ldots n} \geq \Psi_{k}^{k} \Psi_{1 . . \widehat{k} . . n}^{1 . \widehat{k} . . n}, \quad(1 \leq k \leq n)
$$

which imply the following inequality

$$
\left(\Psi_{1 \ldots n}^{1 \ldots n}\right)^{n} \geq \prod_{k=1}^{n} \Psi_{k}^{k} \prod_{k=1}^{n} \Psi_{1 . . . k . . n}^{1 . . \widehat{k} . n}
$$

By Partition property we also have the following inequality

$$
\Psi_{1 \ldots n}^{1 \ldots n} \geq \prod_{k=1}^{n} \Psi_{k}^{k}
$$


The last two inequalities suggest the validity of the following inequality

$$
\left(\Psi_{1 \ldots n}^{1 \ldots n}\right)^{n-1} \geq \prod_{k=1}^{n} \Psi_{1 . . \widehat{k} . . n}^{1 . \widehat{k} . n}
$$

which is far from obvious (see Conjecture 1.5 below) although it would be a simple consequence of our Weighted Multiset Partition Conjecture.

This last conjectural inequality is interesting because it generalizes some special cases of not yet proven conjectures of Atiyah and Sutcliffe on configurations of points in three dimensional Euclidean space.

Our conjecture reads as follows:

Conjecture 1.5 For any $n \geq 1$, let $X_{1} \geq X_{2} \geq \ldots \geq X_{n} \geq 0, \xi_{1}, \xi_{2}, \ldots, \xi_{n} \geq 0$, be nonnegative real numbers. Then we have coefficientwise (w.r.t. $\xi_{1}, \xi_{2}, \ldots, \xi_{n}$ ) inequality

$$
\left(\Psi_{12 \cdots n}^{12 \cdots n}\right)^{n-1} \geq \prod_{k=1}^{n} \Psi_{12 \cdots \hat{k} \cdots n}^{12 \cdots \hat{k} \cdots n}
$$

where $12 \cdots \hat{k} \cdots n$ denotes the sequence $12 \cdots(k-1)(k+1) \cdots n$. The equality obviously holds true iff $X_{1}=X_{2}=\cdots=X_{n}$.

This Conjecture implies the strongest Atiyah-Sutcliffe's conjecture for almost collinear configurations of points (all but one point are collinear, called type(A) in $[6])$.

To illustrate the Conjecture (1.5) we consider first the cases $n=2$ and $n=3$.

Case $n=2$ : We have

$$
\begin{aligned}
\Psi_{12}^{12} & =1+\left(\xi_{1}+\xi_{2}\right) X_{1}+\xi_{1} \xi_{2} X_{1} X_{2}= \\
& =1+\xi_{1} X_{1}+\xi_{2} X_{2}+\xi_{1} \xi_{2} X_{1} X_{2}+\left(X_{1}-X_{2}\right) \xi_{2}= \\
& =\left(1+\xi_{1} X_{1}\right)\left(1+\xi_{2} X_{2}\right)+\xi_{2}\left(X_{1}-X_{2}\right) \geq \\
& \geq\left(1+\xi_{1} X_{1}\right)\left(1+\xi_{2} X_{2}\right)=\Psi_{1}^{1} \Psi_{2}^{2} .
\end{aligned}
$$

Case $n=3$ : We first write $\Psi_{123}^{123}$ in two different ways:

$$
\Psi_{123}^{123}=\xi_{2}\left(X_{1}-X_{2}\right)+\widehat{\Psi}_{1 \underline{2} 3}^{123} \text { and } \Psi_{123}^{123}=\xi_{3}\left(X_{1}-X_{2}\right)+\widehat{\Psi}_{12 \underline{3}}^{123} .
$$


Note that $\widehat{\Psi}_{123}^{123}$ is obtained from $\Psi_{123}^{123}$ by replacing the linear term $\xi_{2} X_{1}$ by $\xi_{2} X_{2}$, hence all its coefficients are nonnegative.

The left hand side of the Conjecture (1.5) $L_{3}$ can be rewritten as follows:

$$
\begin{aligned}
L_{3}=\left(\Psi_{123}^{123}\right)^{2} & =\left(\xi_{2}\left(X_{1}-X_{2}\right)+\widehat{\Psi}_{123}^{123}\right) \Psi_{123}^{123} \\
& =\xi_{2}\left(X_{1}-X_{2}\right) \Psi_{123}^{123}+\widehat{\Psi}_{123}^{123} \Psi_{123}^{123} \\
& =\xi_{2}\left(X_{1}-X_{2}\right) \Psi_{123}^{123}+\widehat{\Psi}_{123}^{123}\left(\xi_{3}\left(X_{1}-X_{2}\right)+\widehat{\Psi}_{12 \underline{3}}^{123}\right) \\
& =L_{3}^{\prime}\left(X_{1}-X_{2}\right)+\widehat{\Psi}_{1 \underline{2} 3}^{123} \widehat{\Psi}_{12 \underline{3}}^{123}
\end{aligned}
$$

where $L_{3}^{\prime}=\xi_{2} \Psi_{123}^{123}+\xi_{3} \widehat{\Psi}_{123}^{123}$ is a positive polynomial.

Now we have

$$
L_{3} \geq \widehat{L}_{3}:=\widehat{\Psi}_{123}^{123} \widehat{\Psi}_{12 \underline{3}}^{123} .
$$

By using the formula

$$
\widehat{\Psi}_{123}^{123}=\Psi_{13}^{12}+\xi_{2} X_{2} \Psi_{13}^{13}=\left(\Psi_{2}^{2}-1\right) \Psi_{13}^{13}+\Psi_{13}^{12}
$$

we can rewrite $\widehat{L}_{3}$ as

$$
\begin{aligned}
\widehat{L}_{3} & =\left[\left(\Psi_{13}^{12}-\Psi_{13}^{13}\right)+\Psi_{2}^{2} \Psi_{13}^{13}\right] \widehat{\Psi}_{12 \underline{3}}^{123} \\
& =\xi_{1} \xi_{3} X_{1}\left(X_{2}-X_{3}\right) \widehat{\Psi}_{12 \underline{3}}^{123}+\Psi_{13}^{13}\left(\Psi_{2}^{2} \widehat{\Psi}_{12 \underline{3}}^{123}\right)
\end{aligned}
$$

The last term in parenthesis can be written as

$$
\begin{aligned}
\Psi_{2}^{2} \widehat{\Psi}_{12 \underline{3}}^{123} & =\Psi_{12}^{12} \Psi_{23}^{23}+\Psi_{2}^{1}\left(\Psi_{23}^{22}-\Psi_{23}^{23}\right) \\
& =\Psi_{12}^{12} \Psi_{23}^{23}+\xi_{2} \xi_{3} X_{2}\left(X_{2}-X_{3}\right) \Psi_{2}^{1},
\end{aligned}
$$

so we get

$$
\widehat{L}_{3}=L_{3}^{\prime \prime}\left(X_{2}-X_{3}\right)+\Psi_{12}^{12} \Psi_{13}^{13} \Psi_{23}^{23}
$$

where $L_{3}^{\prime \prime}$ denotes the positive polynomial

$$
L_{3}^{\prime \prime}=\xi_{1} \xi_{3} X_{1} \widehat{\Psi}_{12 \underline{3}}^{123}+\xi_{2} \xi_{3} X_{2} \Psi_{2}^{1} \Psi_{13}^{13} .
$$

We now have an explicit formula for $L_{3}$ :

$$
L_{3}=L_{3}^{\prime}\left(X_{1}-X_{2}\right)+L_{3}^{\prime \prime}\left(X_{2}-X_{3}\right)+\Psi_{12}^{12} \Psi_{13}^{13} \Psi_{23}^{23}
$$


with $L_{3}^{\prime}, L_{3}^{\prime \prime}$ positive polynomials, which together with $X_{1} \geq X_{2} \geq X_{3}(\geq 0)$ implies that

$$
L_{3} \geq R_{3}:=\Psi_{12}^{12} \Psi_{13}^{13} \Psi_{23}^{23}
$$

and the Conjecture (1.5) $(n=3)$ is proved.

In fact we have proven an instance $n=3 \widehat{L}_{3} \geq R_{3}$ of a stronger conjecture which we are going to formulate now. Let $2 \leq k \leq n$. We define the modified polynomials $\widehat{\Psi}_{12 \ldots k \ldots n}^{12 \ldots k \ldots n}$ as follows:

$$
\widehat{\Psi}_{12 \ldots k \ldots n}^{12 \ldots k \ldots n}:=\xi_{k}\left(X_{2}-X_{1}\right)+\Psi_{12 \ldots n}^{12 \ldots n}
$$

obtained from $\Psi_{12 \ldots n}^{12 \ldots n}$ by replacing only one term $\xi_{k} X_{1}$ by $\xi_{k} X_{2}$, hence $\widehat{\Psi}_{12 \ldots . k n}^{12 \ldots k \ldots n}$ are still positive. Let us introduce the following notation:

$$
\widehat{L}_{n}:=\prod_{k=2}^{n} \widehat{\Psi}_{12 \ldots \underline{k} \ldots n}^{12 \ldots k \ldots n} ; \quad R_{n}:=\prod_{k=1}^{n} \Psi_{12 \ldots \hat{k} \ldots . .}^{12 \ldots \hat{k}} .
$$

Then clearly $L_{n}:=\left(\Psi_{12 \ldots n}^{12 \ldots n}\right)^{n-1} \geq \widehat{L}_{n}$. Now our stronger conjecture reads as

\section{Conjecture 1.6}

$$
\widehat{L}_{n} \geq R_{n}(n \geq 1)
$$

with equality iff $X_{2}=X_{3}=\cdots=X_{n}$.

More generally, we conjecture that the difference $\widehat{L}_{n}-R_{n}$ is a polynomial in the differences $X_{2}-X_{3}, X_{3}-X_{4}, \ldots, X_{n-1}-X_{n}$ with coefficients in $\mathbb{Z}_{\geq 0}\left[X_{1}, \ldots, X_{n}, \xi_{1}, \ldots, \xi_{n}\right]$.

\section{Proposition 1.7}

$$
L_{n}=L_{n}^{\prime}\left(X_{1}-X_{2}\right)+\widehat{L}_{n}
$$

for some positive polynomial $L_{n}^{\prime}$.

\section{Proof of Proposition 1.7.}




$$
\begin{aligned}
& L_{n}=\left(\Psi_{12 \cdots n}^{12 \cdots n}\right)^{n-1}=\left(\xi_{2}\left(X_{1}-X_{2}\right)+\widehat{\Psi}_{12}^{12 \cdots n}\right)\left(\Psi_{12 \cdots n}^{12 \cdots n}\right)^{n-2} \\
& =\xi_{2}\left(X_{1}-X_{2}\right)\left(\Psi_{12 \cdots n}^{12 \cdots n}\right)^{n-2}+\widehat{\Psi}_{12 \cdots n}^{12 \cdots n}\left(\xi_{3}\left(X_{1}-X_{2}\right)+\widehat{\Psi}_{123 \cdots n}^{123 \cdots n}\right)\left(\Psi_{12 \cdots n}^{12 \cdots n}\right)^{n-3} \\
& =\xi_{2}\left(X_{1}-X_{2}\right)\left(\Psi_{12 \cdots n}^{12 \cdots n}\right)^{n-2}+\xi_{3}\left(X_{1}-X_{2}\right) \widehat{\Psi}_{12 \cdots n}^{12 \cdots n}\left(\Psi_{12 \cdots n}^{12 \cdots n}\right)^{n-3}+ \\
& +\widehat{\Psi}_{12 \cdots n}^{12 \cdots n} \widehat{\Psi}_{12 \underline{3} \cdots n}^{123 \cdots n}\left(\Psi_{12 \cdots n}^{12 \cdots n}\right)^{n-3} \\
& \text { : } \\
& =\left(\sum_{k=1}^{n-1} \xi_{k+1}\left(\prod_{j=2}^{k} \widehat{\Psi}_{12 \ldots j \ldots n}^{12 \ldots j \ldots n}\right)\left(\Psi_{12 \ldots n}^{12 \ldots n}\right)^{n-k}\right)\left(X_{1}-X_{2}\right)+\prod_{j=2}^{n} \widehat{\Psi}_{12 \ldots j \ldots n}^{12 \ldots j \ldots n} .
\end{aligned}
$$

Now we turn to study the quotient

$$
\frac{L_{n}}{R_{n}}=\frac{\left(\Psi_{1 \ldots n}^{1 \ldots n}\right)^{n-1}}{\prod_{k=1}^{n} \Psi_{1 \ldots \widehat{k} \ldots n}^{1 \ldots \widehat{k} \ldots n}}
$$

by studying the growth behaviour of quotients of its factors $\Psi_{1 \ldots n}^{1 \ldots n} / \Psi_{11 \ldots \widehat{k} \ldots n}^{1 \ldots \widehat{k} \ldots n}$ w.r.t. any of its arguments $X_{r}, 1 \leq r \leq n$.

In the following theorem we obtain an explicit formula for the numerators of the derivatives w.r.t. $X_{r},(1 \leq r \leq n, r \neq k)$ of the quantities $\Psi_{1 \ldots n}^{1 \ldots n} / \Psi_{1 \ldots \widehat{k} \ldots . .}^{1 \ldots \widehat{k}}$. From this formulas we get some monotonicity properties which enable us to state some new (refined) conjectures later on.

Theorem 1.8 Let

$$
\Delta_{r}:=\partial_{X_{r}} \Psi_{1 \ldots n}^{1 \ldots n} \cdot \Psi_{1 \ldots \widehat{k} \ldots n}^{1 \ldots \widehat{k} \ldots n}-\Psi_{1 \ldots n}^{1 \ldots n} \cdot \partial_{X_{r}} \Psi_{1 \ldots \widehat{k} \ldots n}^{1 \ldots \widehat{k} \ldots n}, \quad(1 \leq r \leq n) .
$$

Then we have the following explicit formulas

(i) for any $r, 1 \leq r<k(\leq n)$ we have

$$
\begin{aligned}
\Delta_{r}= & \xi_{k} \sum_{0 \leq i<r \leq j \leq n} s_{(j-1, i)^{\prime}}^{(k)} X_{1}^{2} \cdots X_{i}^{2} X_{i+1} \cdots \widehat{X}_{k} \cdots X_{j}+ \\
& +\sum_{0 \leq i<r, k \leq j<n} e_{i} e_{j}^{(k)} X_{1}^{2} \cdots X_{i}^{2} X_{i+1} \cdots \widehat{X}_{r} \cdots \widehat{X}_{k} \cdots X_{j}\left(X_{k}-X_{j+1}\right) \\
&
\end{aligned}
$$


(ii) for any $r,(1 \leq) k<r \leq n$ we have

$$
\begin{aligned}
\Delta_{r}= & -\left(\sum_{\substack{0 \leq i<r \leq j \leq n \\
(k-1, i)^{\prime}}} X_{1}^{2} \cdots X_{i}^{2} X_{i+1} \cdots \widehat{X}_{k} \cdots \widehat{X}_{r} \cdots X_{j}+\right. \\
& \left.+\sum_{0 \leq i<k, r \leq j<n} e_{i}^{(k)} e_{j} X_{1}^{2} \cdots X_{i}^{2} X_{i+1} \cdots \widehat{X}_{k} \cdots \widehat{X}_{r} \cdots X_{j}\left(X_{j+1}-X_{k}\right)\right)
\end{aligned}
$$

where $s_{\lambda}^{(k)}$ denotes the $\lambda$-th Schur function of $\xi_{1}, \ldots, \xi_{k-1}, \xi_{k+1}, \ldots, \xi_{n}\left(\xi_{k}\right.$ omitted).

\section{Proof of Theorem 1.8.}

(i) For any $r, 1 \leq r<k(\leq n)$ we find explicitly a formula as follows. We shall use notations $X_{1 . . i}:=X_{1} X_{2} \cdots X_{i}$, for multilinear monomials and $e_{i}:=e_{i}\left(\xi_{1}, \ldots, \xi_{n}\right)$, $e_{i}^{(k)}=e_{i}\left(\xi_{1}, \ldots, \widehat{\xi}_{k}, \ldots \xi_{n}\right)$ for the elementary symmetric functions (here $k$ is fixed). Then we can rewrite our basic quantities

$$
\begin{aligned}
\Psi_{1 \ldots n}^{1 \ldots n}:=\sum_{i=0}^{n} e_{i} X_{1 . . i} & \\
\Psi_{1 \ldots \widehat{k} \ldots . . . n}^{1 \ldots \widehat{k}} & :=\sum_{i=0}^{k-1} e_{i}^{(k)} X_{1 . . i}+\frac{1}{X_{k}} \sum_{i=k}^{n-1} e_{i}^{(k)} X_{1 . . i+1}= \\
& =\sum_{i=0}^{n-1} e_{i}^{(k)} X_{1 . . i}+\frac{1}{X_{k}} \sum_{i=k}^{n-1} e_{i}^{(k)} X_{1 . . i}\left(X_{i+1}-X_{k}\right)
\end{aligned}
$$

For the derivatives we get immediately

$$
\begin{gathered}
\partial_{X_{r}} \Psi_{1 \ldots n}^{1 \ldots n}=\frac{1}{X_{r}} \sum_{i=r}^{n} e_{i} X_{1 . . i}=\frac{1}{X_{r}}\left(\Psi_{1 \ldots n}^{1 \ldots n}-\sum_{i=0}^{r-1} e_{i} X_{1 . . i}\right) \\
\partial_{X_{r}} \Psi_{1 \ldots \widehat{k} \ldots n}^{1 \ldots \widehat{k} \ldots n}=\frac{1}{X_{r}} \sum_{i=r}^{n-1} e_{i}^{(k)} X_{1 . . i}+\frac{1}{X_{k} X_{r}} \sum_{i=k}^{n-1} e_{i}^{(k)} X_{1 . . i}\left(X_{i+1}-X_{k}\right) \\
=\frac{1}{X_{r}}\left(\Psi_{1 \ldots \widehat{k} \ldots n}^{1 \ldots \widehat{k} \ldots n}-\sum_{i=0}^{r-1} e_{i}^{(k)} X_{1 . . i}\right)
\end{gathered}
$$


By plugging (1.6) and (1.8) into (1.3) we obtain

$$
X_{r} \Delta_{r}=\Psi_{1 \ldots n}^{1 \ldots n}\left(\sum_{i=0}^{r-1} e_{i}^{(k)} X_{1 . . i}\right)-\Psi_{1 \ldots \widehat{k} \ldots n}^{1 \ldots \widehat{k} \ldots n}\left(\sum_{i=0}^{r-1} e_{i} X_{1 . . i}\right)=
$$

and after simple cancelation, by invoking (1.5) we get

$$
\begin{aligned}
= & \left(\sum_{j=r}^{n} e_{j} X_{1 . . j}\right)\left(\sum_{i=0}^{r-1} e_{i}^{(k)} X_{1 . . i}\right)- \\
& \quad\left(\sum_{j=r}^{n-1} e_{j}^{(k)} X_{1 . . j}+\frac{1}{X_{k}} \sum_{j=k}^{n-1} e_{j}^{(k)} X_{1 . . j}\left(X_{j+1}-X_{k}\right)\right)\left(\sum_{i=0}^{r-1} e_{i} X_{1 . . i}\right)
\end{aligned}
$$

i.e.

$$
X_{r} \Delta_{r}=\sum_{0 \leq i<r \leq j \leq n}\left(e_{j} e_{i}^{(k)}-e_{i} e_{j}^{(k)}\right) X_{1 . . i} X_{1 . . j}+\frac{1}{X_{k}} \sum_{0 \leq i<r, k \leq j<n} e_{i} e_{j}^{(k)} X_{1 . . i} X_{1 . . j}\left(X_{k}-X_{j+1}\right)
$$

If we use a simple identity $e_{j}=e_{j}^{(k)}+\xi_{k} e_{j-1}^{(k)}$, we can identify the quantity

$$
\begin{aligned}
e_{j} e_{i}^{(k)}-e_{i} e_{j}^{(k)} & =\left(e_{j}^{(k)}+\xi_{k} e_{j-1}^{(k)}\right) e_{i}^{(k)}-\left(e_{i}^{(k)}+\xi_{k} e_{i-1}^{(k)}\right) e_{j}^{(k)}= \\
& =\left|\begin{array}{cc}
e_{j-1}^{(k)} & e_{j}^{(k)} \\
e_{i-1}^{(k)} & e_{i}^{(k)}
\end{array}\right| \xi_{k}=s_{2^{i} 1^{j-i-1}}^{(k)} \xi_{k}
\end{aligned}
$$

Thus in this case $(1 \leq r<k)$ we obtain a formula

$$
\begin{aligned}
\Delta_{r}=\xi_{k} \sum_{0 \leq i<r \leq j \leq n} & s_{(j-1, i)^{\prime}}^{(k)} X_{1}^{2} \cdots X_{i}^{2} X_{i+1} \cdots \widehat{X}_{r} \cdots X_{j}+ \\
& +\sum_{0 \leq i<r, k \leq j<n} e_{i} e_{j}^{(k)} X_{1}^{2} \cdots X_{i}^{2} X_{i+1} \cdots \widehat{X}_{r} \cdots \widehat{X}_{k} \cdots X_{j}\left(X_{k}-X_{j+1}\right)
\end{aligned}
$$

(where $e_{j}^{(k)}=e_{j}\left(\xi_{1}, \ldots, \widehat{\xi_{k}}, \ldots, \xi_{n}\right)$ ) in terms of Schur functions (of arguments $\left.\xi_{1}, \ldots, \widehat{\xi_{k}}, \ldots, \xi_{n}\right)$ corresponding to a transpose $(j-1, i)^{\prime}=\left(2^{i} 1^{j-i-1}\right)$ of a partition $(j-1, i)$ (cf. Jacobi-Trudi formula, I 3.5 in [8]).

(ii) For any $r,(1 \leq) k<r \leq n$. In this case we use

$$
\partial_{X_{r}} \Psi_{1 \ldots \widehat{k} \ldots n}^{1 \ldots \widehat{k} \ldots n}=\frac{1}{X_{k} X_{r}} \sum_{j=r-1}^{n-1} e_{j}^{(k)} X_{1 . . j+1}
$$




$$
\begin{aligned}
\Psi_{1 \ldots \widehat{k} \ldots n}^{1 \ldots \widehat{k} \ldots n} & =\sum_{i=0}^{k-1} e_{i}^{(k)} X_{1 . . i}+\frac{1}{X_{k}} \sum_{i=k}^{n-1} e_{i}^{(k)} X_{1 . . i+1}= \\
& =\frac{1}{X_{k}}\left(\sum_{i=0}^{k-1} X_{1 . . i}\left(X_{k}-X_{i+1}\right)+\sum_{i=0}^{n-1} e_{i}^{(k)} X_{1 . . i}\right)
\end{aligned}
$$

By plugging this into (1.3) we get

$$
\begin{gathered}
X_{k} X_{r} \Delta_{r}=\left(\sum_{j=r}^{n} e_{j} X_{1 . . j}\right)\left(\sum_{i=0}^{k-1} e_{i}^{(k)} X_{1 . . i}\left(X_{k}-X_{i+1}\right)+\sum_{i=0}^{n-1} e_{i}^{(k)} X_{1 . . i+1}\right)- \\
-\left(\sum_{j=0}^{r-1} e_{j} X_{1 . . j}+\sum_{j=r}^{n} e_{j} X_{1 . . j}\right)\left(\sum_{i=r-1}^{n-1} e_{i}^{(k)} X_{1 . . i+1}\right) \\
=\left(\sum_{i=0}^{r-2} e_{i}^{(k)} X_{1 . . i+1}\right)\left(\sum_{j=r}^{n} e_{j} X_{1 . . j}\right)-\left(\sum_{i=0}^{r-1} e_{i} X_{1 . . i}\right)\left(\sum_{j=r-1}^{n-1} e_{j}^{(k)} X_{1 . . j+1}\right)+ \\
+\sum_{i=0}^{k-1} \sum_{j=r}^{n} e_{i}^{(k)} e_{j} X_{1 . . i} X_{1 . . j}\left(X_{k}-X_{i+1}\right) \\
=\left(\sum_{i=1}^{r-1} e_{i-1}^{(k)} X_{1 . . i}\right)\left(\sum_{j=r}^{n} e_{j} X_{1 . . j}\right)-\left(\sum_{i=0}^{r-1} e_{i} X_{1 . . i}\right)\left(\sum_{j=r}^{n} e_{j-1}^{(k)} X_{1 . . j}\right)+ \\
+\sum_{i=0}^{k-1} \sum_{j=r}^{n} e_{i}^{(k)} e_{j} X_{1 . . i} X_{1 . . j}\left(X_{k}-X_{i+1}\right)
\end{gathered}
$$

By using a formula for elementary symmetric functions $\left(e_{i}=e_{i}^{(k)}+\xi_{k} e_{i-1}^{(k)}\right)$ we can write in terms of Schur functions (of arguments $\xi_{1}, \ldots, \xi_{k-1}, \xi_{k+1}, \ldots, \xi_{n}$ ), where $\lambda^{\prime}$ is a conjugate of $\lambda$.

$$
e_{i-1}^{(k)} e_{j}-e_{i} e_{j-1}^{(k)}=e_{i-1}^{(k)} e_{j}^{(k)}-e_{i}^{(k)} e_{j-1}^{(k)}=-\left|\begin{array}{cc}
e_{j-1}^{(k)} & e_{j}^{(k)} \\
e_{i-1}^{(k)} & e_{i}^{(k)}
\end{array}\right|=-s_{2^{i} 1^{j-i-1}}^{(k)}=-s_{(j-1, i)^{\prime}}^{(k)}
$$

Thus we obtain a formula

$$
\begin{aligned}
\Delta_{r}=-( & \sum_{\substack{0 \leq i<r \leq j \leq n \\
j(j)^{\prime}}} s_{(j)}^{(k)} X_{1}^{2} \cdots X_{i}^{2} X_{i+1} \cdots \widehat{X}_{k} \cdots \widehat{X}_{r} \cdots X_{j}+ \\
& \left.\quad+\sum_{0 \leq i<k, r \leq j<n} e_{i}^{(k)} e_{j} X_{1}^{2} \cdots X_{i}^{2} X_{i+1} \cdots \widehat{X}_{k} \cdots \widehat{X}_{r} \cdots X_{j}\left(X_{j+1}-X_{k}\right)\right)
\end{aligned}
$$


Corollary $1.9\left(X_{r}-\right.$ monotonicity $)$

Let $X_{1} \geq \cdots \geq X_{n} \geq 0, \xi_{1}, \ldots, \xi_{n} \geq 0$ be as before. Then

(i) for any $r, 1 \leq r<k(\leq n)$ we have

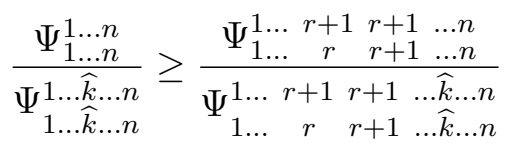

(ii) for any $r,(1 \leq) k<r(\leq n)$ we have

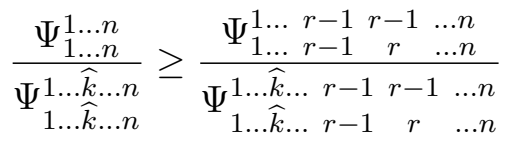

Now we illustrate how to use Corollary 1.9 to prove our Conjecture 1.5 for $n=2,3,4$ and 5 .

Case $n=2$

$$
Q_{2}:=\frac{\Psi_{12}^{12}}{\Psi_{1}^{1} \Psi_{2}^{2}} \geq \frac{\Psi_{12}^{22}}{\Psi_{1}^{2} \Psi_{2}^{2}}=1(\text { by }(i))
$$

Case $n=3$

$$
\begin{aligned}
Q_{3}:=\frac{\Psi_{123}^{123} \Psi_{123}^{123}}{\Psi_{12}^{12} \Psi_{13}^{13} \Psi_{23}^{23}} & \geq \frac{\Psi_{123}^{223} \Psi_{123}^{123}}{\Psi_{12}^{22} \Psi_{13}^{13} \Psi_{23}^{23}} \geq \frac{\Psi_{123}^{223} \Psi_{123}^{223}}{\Psi_{12}^{22} \Psi_{13}^{23} \Psi_{23}^{23}}(\text { by }(i)) \\
& \geq \frac{\Psi_{123}^{222} \Psi_{123}^{223}}{\Psi_{12}^{22} \Psi_{13}^{22} \Psi_{23}^{23}} \geq \frac{\Psi_{123}^{222} \Psi_{123}^{222}}{\Psi_{12}^{22} \Psi_{13}^{22} \Psi_{23}^{22}}=1 \text { (by }(i i) \text { ) }
\end{aligned}
$$

Case $n=4$

$$
Q_{4}:=\frac{\left(\Psi_{1234}^{1234}\right)^{3}}{\Psi_{123}^{123} \Psi_{124}^{124} \Psi_{134}^{134} \Psi_{234}^{234}} \geq \cdots \geq \frac{\Psi_{1234}^{2244}\left(\Psi_{1234}^{2224}\right)^{2}}{\Psi_{123}^{224} \Psi_{124}^{224} \Psi_{134}^{224} \Psi_{234}^{224}} \quad(\geq 1)
$$

This last inequality follows from the following symmetric function identity:

$$
\begin{aligned}
& \Psi_{1234}^{2244}\left(\Psi_{1234}^{2224}\right)^{2}-\Psi_{123}^{224} \Psi_{124}^{224} \Psi_{134}^{224} \Psi_{234}^{224}= \\
& X_{2}^{2} X_{4}^{4} m_{2222}+2 X_{2}^{2} X_{4}^{3} m_{2221}+X_{2}^{2} X_{4}^{2} m_{222}+3 X_{2}^{2} X_{4}^{2} m_{2211}+X_{2}^{2} X_{4} m_{221} \\
& +4 X_{2}^{2} X_{4} m_{2111}+X_{2}^{2} m_{211}+X_{2}\left(3 X_{2}+2 X_{4}\right) m_{1111}+X_{2} m_{111}
\end{aligned}
$$


where $m_{\lambda}=m_{\lambda}\left(\xi_{1}, \xi_{2}, \xi_{3}, \xi_{4}\right)$ are the monomial symmetric functions.

Case $n=5$

$$
Q_{5}:=\frac{\left(\Psi_{1 \ldots .5}^{1 \ldots .5}\right)^{4}}{\prod_{k=1}^{5} \Psi_{1 \ldots . . . . .5}^{11 . . k \ldots 5}} \geq \cdots \geq \frac{\left(\Psi_{12245}^{22244} \Psi_{12345}^{22444}\right)^{2}}{\Psi_{1234}^{2244} \Psi_{1235}^{2244} \Psi_{1245}^{2244} \Psi_{1345}^{2244} \Psi_{2345}^{2244}} \quad(\geq 1)
$$

The last inequality is equivalent to an explicit symmetric function identity with all coefficients (w.r.t. monomial basis) positive.

Now we state our stronger conjecture.

Conjecture $\mathbf{1 . 1 0}$ (for symmetric functions)

Let $X_{1} \geq X_{2} \geq \cdots \geq X_{n} \geq 0$ and $\xi_{1}, \ldots, \xi_{n} \geq 0$. Then the inequalities

(a) For $n$ even

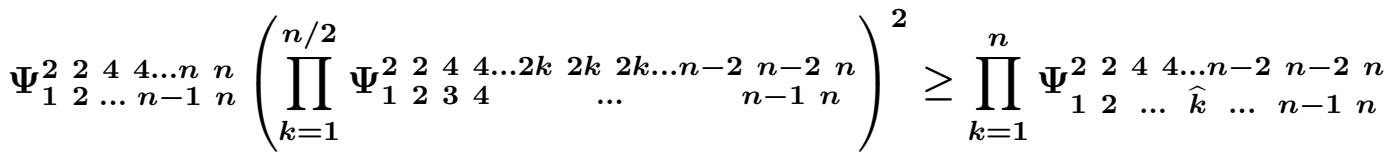

(b) For $n$ odd

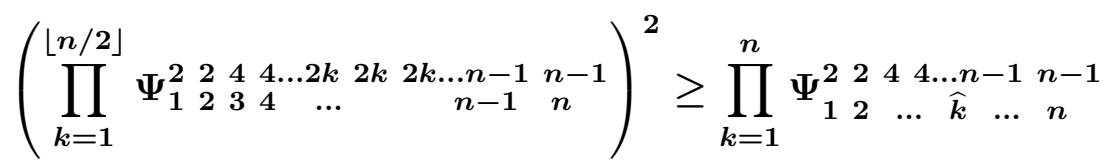

hold true coefficientwise (m-positivity).

Now we motivate another inequalities for symmetric functions which also refine the strongest Atiyah-Sutcliffe conjecture for configurations of type (A). Let $n=3$. We apply Corollary 1.9 by using steps (ii) only.

$$
Q_{3}:=\frac{\Psi_{123}^{123} \Psi_{123}^{123}}{\Psi_{12}^{12} \Psi_{13}^{13} \Psi_{23}^{23}} \geq \frac{\Psi_{123}^{113} \Psi_{123}^{123}}{\Psi_{12}^{12} \Psi_{13}^{13} \Psi_{23}^{13}} \geq \frac{\Psi_{123}^{112} \Psi_{123}^{123}}{\Psi_{12}^{12} \Psi_{13}^{12} \Psi_{23}^{13}} \geq \frac{\Psi_{123}^{112} \Psi_{123}^{122}}{\Psi_{12}^{12} \Psi_{13}^{12} \Psi_{23}^{12}} \geq 1
$$

The last inequality is equivalent to nonnegativity of the expression

$$
\Psi_{123}^{112} \Psi_{123}^{122}-\Psi_{12}^{12} \Psi_{13}^{12} \Psi_{23}^{12} \quad\left(=X_{1}\left(X_{1}-X_{2}\right)^{2} \xi_{1} \xi_{2} \xi_{3} \geq 0\right) .
$$

Similarly, for $n=4$, the symmetric function inequality stronger than $Q_{4} \geq 1$ would be the following

$$
\Psi_{1234}^{1123} \Psi_{1234}^{1223} \Psi_{1234}^{1233} \geq \Psi_{123}^{123} \Psi_{124}^{123} \Psi_{134}^{123} \Psi_{234}^{123}
$$

Now we state a general conjecture for symmetric functions which imply the strongest Atiyah-Sutcliffe conjecture for almost collinear type (A) configurations. 
Conjecture 1.11 Let $X_{1} \geq \cdots \geq X_{n} \geq, \xi_{1}, \ldots \xi_{n} \geq 0$. Then the following inequality for symmetric functions in $\xi_{1}, \ldots, \xi_{n}$

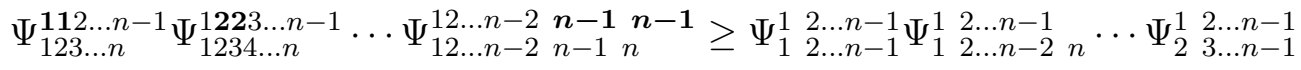

i.e.

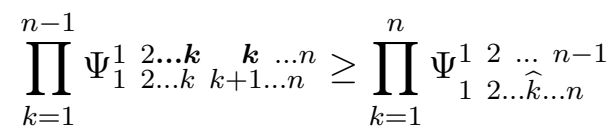

holds true coefficientwise (m-positivity, even s-positivity).

Remark 1.12 Conjectures 1.10 and 1.11 seems to hold also for the Schur basis of symmetric functions in $\xi_{1}, \ldots, \xi_{n}$.

We have checked this Conjecture 1.11 up to $n=5$ by using Maple and symmetric function package SF of J. Stembridge. For $n$ bigger than five the computations are extremely intensive and hopefully in the near future would be possible by using more powerful computers.

Note that the right hand side of the Conjecture 1.11 involves symmetric functions of partial alphabets $\xi_{1}, \xi_{2}, \ldots, \xi_{k-1}, \xi_{k+1}, \ldots, \xi_{n}$. But the left hand side doesn't have this "defect". Our objective now is to give explicit formula for the right hand side in terms of the elementary symmetric functions of the full alphabet $\xi_{1}, \xi_{2}, \ldots, \xi_{n}$. This we are going to achieve by using resultants as follows.

Lemma 1.13 For any $k,(1 \leq k \leq n)$, we have

$$
\Psi_{1 \ldots \widehat{k} \ldots n}^{1 \ldots k \ldots n-1}=\sum_{j=0}^{n-1} a_{j} \xi_{k}^{n-1-j}
$$

where

$$
\begin{aligned}
& a_{n-1}=1+X_{1} e_{1}+X_{1} X_{2} e_{2}+\ldots+X_{1} \cdots X_{n-1} e_{n-1}, \\
& a_{n-2}=-X_{1}-X_{1} X_{2} e_{1}-\ldots-X_{1} \cdots X_{n-1} e_{n-2}, \\
& \cdots \\
& a_{0}=(-1)^{n-1} X_{1} \cdots X_{n-1} \\
& \text { i.e. } \\
& a_{n-1-j}=(-1)^{j} \sum_{i=j}^{n-1} X_{1} \cdots X_{i} e_{i-j}
\end{aligned}
$$


Proof of Lemma 1.13.

By definition we have

$$
\Psi_{1 \ldots \widehat{k} \ldots n}^{1 \ldots n-1}=\sum_{i=0}^{n-1} X_{1} \cdots X_{i} e_{i}^{(k)}
$$

where $e_{i}^{(k)}$ is the $i$-th elementary function of $\xi_{1}, \ldots, \xi_{k-1}, \xi_{k+1}, \ldots, \xi_{n}$. Now from the decomposition

$$
\left(1+\xi_{k} t\right)^{-1} \prod_{j=1}^{n}\left(1+\xi_{j} t\right)=\prod_{j \neq k}\left(1+\xi_{j} t\right)=\sum_{i=0}^{n-1} e_{i}^{(k)} t^{i}
$$

we get

$$
e_{i}^{(k)}=e_{i}-e_{i-1} \xi_{k}+e_{i-2} \xi_{k}^{2}-\cdots+(-1)^{i} \xi_{k}^{i}
$$

By substituting this into equation (1.9) the Lemma 1.13 follows.

Then, by Lemma 1.13, the right hand side of the Conjecture 1.11

$$
R_{n}=\prod_{k=1}^{n} \begin{array}{cccccc}
\Psi_{1}^{1} & 2 & \ldots & k & \ldots & n-1 \\
1 & 2 & \ldots & \widehat{k} & \ldots & n
\end{array}=\prod_{k=1}^{n}\left(\sum_{j=0}^{n-1} a_{j} \xi_{k}^{n-1-j}\right)
$$

can be interpreted as a resultant $R_{n}=\operatorname{Resultant}(f, g)$ of the following two polynomials

$$
\begin{aligned}
f(x) & =\sum_{j=0}^{n-1} a_{j} x^{n-1-j} \\
g(x) & =\prod_{i=1}^{n}\left(x-\xi_{i}\right)=\sum_{j=0}^{n}(-1)^{j} e_{j} x^{n-j}
\end{aligned}
$$


Then Sylvester formula

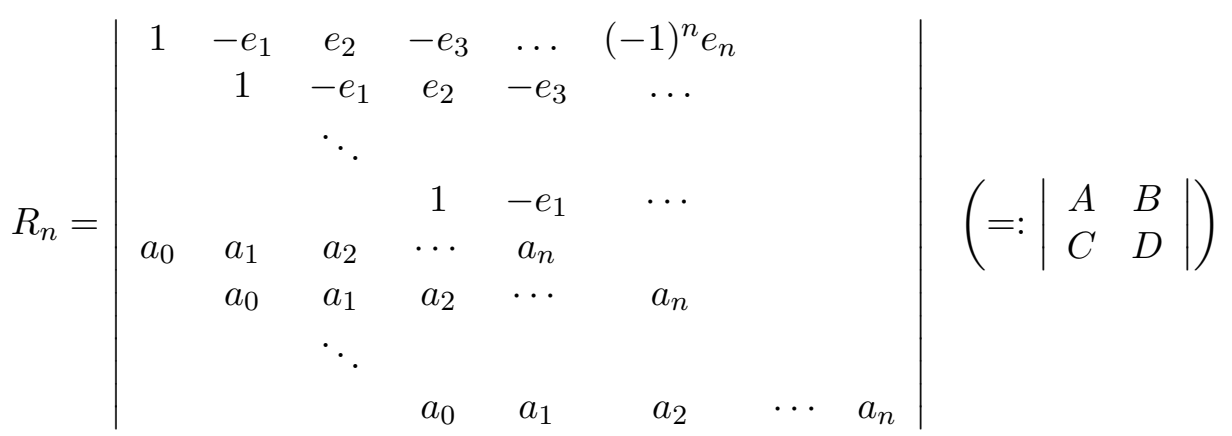

can be simplified as

$$
=|A| \cdot\left|D-C A^{-1} B\right|=\left|D-C A^{-1} B\right| .
$$

The entries of the $n \times n$ matrix $\Delta:=D-C A^{-1} B$ are given by

$$
\delta_{i j}= \begin{cases}(-1)^{j-i-1} \sum_{k=j+1}^{n} X_{1} \cdots X_{k+i-j} e_{k}, & 0 \leq i<j \leq n-1 \\ (-1)^{j-i} \sum_{k=0}^{j} X_{1} \cdots X_{k+i-j} e_{k}, & 0 \leq j \leq i \leq n-1\end{cases}
$$

For example, for $n=3$

$$
\Delta_{3}=\left|\begin{array}{ccc}
1 & X_{1} e_{2}+X_{1} X_{2} e_{3} & -X_{1} e_{3} \\
-X_{1} & 1+X_{1} e_{1} & X_{1} X_{2} e_{3} \\
X_{1} X_{2} & -X_{1}-X_{1} X_{2} e_{1} & 1+X_{1} e_{1}+X_{1} X_{2} e_{2}
\end{array}\right|
$$

By elementary operations (including multiplication of $2^{\text {nd }}$ row and column by -1 ) we get

$$
\begin{aligned}
\Delta_{3} & =\left|\begin{array}{ccc}
1 & * & * \\
0 & \Psi_{123}^{112} & X_{1}\left(X_{2}-X_{1}\right) e_{3} \\
0 & X_{2}-X_{1} & \Psi_{123}^{122}
\end{array}\right|=\left|\begin{array}{cc}
\Psi_{123}^{112} & X_{1}\left(X_{2}-X_{1}\right) e_{3} \\
X_{2}-X_{1} & \Psi_{123}^{122}
\end{array}\right|= \\
& =\left|\begin{array}{cc}
\Psi_{123}^{112} & X_{1}\left(X_{1}-X_{2}\right) e_{3} \\
X_{1}-X_{2} & \Psi_{123}^{122}
\end{array}\right| .
\end{aligned}
$$


Similarly, for $n=4$ we obtain

$$
\begin{aligned}
\Delta_{4} & =\left|\begin{array}{ccc}
\Psi_{1234}^{1123} & -X_{1}\left(X_{1}-X_{2}\right) e_{3}-X_{1} X_{2}\left(X_{1}-X_{3}\right) e_{4} & X_{1}\left(X_{1}-X_{2}\right) e_{4} \\
-\left(X_{1}-X_{2}\right) & \Psi_{1234}^{1223} & -X_{1} X_{2}\left(X_{2}-X_{3}\right) e_{4} \\
X_{1}\left(X_{2}-X_{3}\right) & -\left(X_{1}-X_{3}\right)-X_{1}\left(X_{2}-X_{3}\right) e_{1} & \Psi_{1234}^{1233}
\end{array}\right|= \\
& =\left|\begin{array}{ccc}
\Psi_{1234}^{1123} & X_{1}\left(X_{1}-X_{2}\right) e_{3}+X_{1} X_{2}\left(X_{1}-X_{3}\right) e_{4} & X_{1}\left(X_{1}-X_{2}\right) e_{4} \\
\left(X_{1}-X_{2}\right) & \Psi_{1234}^{1223} & X_{1} X_{2}\left(X_{2}-X_{3}\right) e_{4} \\
X_{1}\left(X_{2}-X_{3}\right) & \left(X_{1}-X_{3}\right)+X_{1}\left(X_{2}-X_{3}\right) e_{1} & \Psi_{1234}^{1233}
\end{array}\right| .
\end{aligned}
$$

By using abbreviations $X_{i j}=X_{i}-X_{j}$ it can be rewritten as

$$
\Delta_{4}=\left|\begin{array}{ccc}
\Psi_{1234}^{1123} & X_{1} X_{12} e_{3}+X_{1} X_{2} X_{13} e_{4} & X_{1} X_{12} e_{4} \\
X_{12} & \Psi_{1234}^{1223} & X_{1} X_{2} X_{23} e_{4} \\
X_{1} X_{23} & X_{13}+X_{1} X_{23} e_{1} & \Psi_{1234}^{1233}
\end{array}\right| .
$$

In general

$$
\Delta_{n}=\operatorname{det}\left(\delta_{i j}^{\prime}\right)_{1 \leq i, j \leq n-1}
$$

where

$$
\delta_{i j}^{\prime}= \begin{cases}\sum_{k=j+1}^{n} X_{1} \cdots X_{k+i-j-1}\left(X_{i}-X_{k+i-j}\right) e_{k} & , 1 \leq i<j \leq n-1 \\ \Psi_{1}^{1} \ldots \ldots i \ldots n & , i=j \\ \sum_{k=0}^{j} X_{1} \cdots X_{k+i-j-1}\left(X_{k+i-j}-X_{i}\right) e_{k} & , 1 \leq j<i \leq n-1\end{cases}
$$

Note that all $\delta_{i j}^{\prime}$ are symmetric polynomials with non negative coefficients.

Corollary 1.14 The conjecture 1.11 is equivalent to a Hadamard type inequality, holding coefficientwise (m-positivity, even s-positivity), for the (non Hermitian) matrix $\left(\delta_{i j}^{\prime}\right)_{1 \leq i, j \leq n-1}$, i.e.

$$
\prod_{i=1}^{n-1} \delta_{i i}^{\prime} \geq \operatorname{det}\left(\delta_{i j}^{\prime}\right)
$$




\section{Verification of the Đoković's strengthening of the Atiyah- Sutcliffe Conjecture (C2) for some nonplanar config- urations with dihedral symmetry}

Here we basically follow Đoković's [7], where he considered the following dihedral configurations $C_{m, n}$ (type $D$ ).

Let $N=m+n$ points be such that

1. The first $m$ points $x_{1}, \ldots, x_{m}$ lie on a line $L$.

2. The remaining $n$ points $y_{j}=x_{m+j+1}(j=0,1, \ldots, n-1)$ are the vertices of a regular $n$-gon whose plane is perpendicular to $L$ and whose centroid lies on $L$.

He only proved Atiyah conjecture C1. We make some additional refinements including a proof of Atiyah-Sutcliffe conjecture C2 for such configurations.

We may assume $L=\mathbb{R} \times\{0\} \subset \mathbb{R} \times \mathbb{C}=\mathbb{R}^{3}$ and write $x_{i}=\left(a_{i}, 0\right), 1 \leq i \leq m$, $a_{1} \leq \ldots \leq a_{m}$ and $y_{j}=\left(0, b_{j}\right), b_{j}=-\xi^{j}, \xi=e^{2 \pi i / n}, 0 \leq j \leq n-1$.

We set

$$
\lambda_{i}=a_{i}+\sqrt{1+a_{i}^{2}}
$$

Recall that $a_{1}<\cdots<a_{m}$ and, consequently $0<\lambda_{1}<\cdots<\lambda_{m}$. Then the associated polynomials $p_{i}$ (up to scalar factors) are given by

$$
\begin{aligned}
& p_{i}(x, y)=x^{m-i} y^{i-1}\left(x^{n}-\lambda_{i}^{n} y^{n}\right), 1 \leq i \leq m \\
& p_{m+j+1}(x, y)=\prod_{s \neq j}\left(x+\frac{\overline{b_{s}}-\overline{b_{j}}}{\left|b_{s}-b_{j}\right|} y\right) \cdot \prod_{i=1}^{m}\left(y-\lambda_{i} b_{j} x\right), 0 \leq j<n
\end{aligned}
$$

By noting that

$$
b_{s}-b_{j}=2 i \xi^{\frac{j+s}{2}} \sin \frac{\pi(j-s)}{n}
$$

(in Đoković $\xi^{j+s}$ should be replaced by $\xi^{\frac{j+s}{2}}$ ) we obtain

$$
x+\frac{\overline{b_{s}}-\overline{b_{j}}}{\left|b_{s}-b_{j}\right|} y=\left(-\overline{b_{j}} y-i \xi^{\frac{s-j}{2}} \operatorname{sgn}(s-j)\right) \frac{1-\overline{b_{s}} b_{j}}{\left|b_{s}-b_{j}\right|}
$$


and

$$
y-\lambda_{i} b_{j} x=-b_{j}\left(-\overline{b_{j}} y+\lambda_{i} x\right)
$$

Note also that

$$
\left\{\xi^{\frac{s-j}{2}} \operatorname{sgn}(s-j) \mid s=1, \ldots, j-1, j+1, \ldots, n\right\}=\left\{e^{\pi i k / n} \mid k=1, \ldots, n-1\right\}
$$

Thus, after dehomogenizing the polynomials $p_{i}$ by setting $x=1$, we obtain (up to scalar factors) the following polynomials:

$$
\begin{aligned}
& \widetilde{P}_{i}(y)=y^{i-1}\left(1-\lambda_{i}^{n} y^{n}\right), 1 \leq i \leq m \\
& \widetilde{P}_{m+j+1}(y)=f\left(\xi^{-1} y\right), 0 \leq j<n
\end{aligned}
$$

where

$$
f(y)=\prod_{s=1}^{n-1}\left(y-i e^{\pi i s / n}\right) \prod_{i=1}^{m}\left(y+\lambda_{i}\right)
$$

(in Đoković the last $n$ polynomials are reordered)

The main result of Đokovic is the Theorem 3.1 where he proved Atiyah conjecture for configurations described above, by explicitly computing the determinant of the coefficients matrix $\widetilde{P}$ of the polynomials $\{\widetilde{p}_{k}(y) \mid k=1, \ldots, \underbrace{m+n}_{N}\}$ in terms of the coefficients of

$$
f(y)=\sum_{k=0}^{N-1} \widetilde{E}_{k} y^{N-1-k}
$$

His formula reads as follows:

$$
|\operatorname{det}(\widetilde{P})|=n^{n / 2} \prod_{k=0}^{n-1} f_{k}
$$

where

$$
f_{k}=\sum_{s \geq 0}\left(\prod_{j=1}^{s} \lambda_{N-j n-k}^{n}\right) \widetilde{E}_{k+s n}, 0 \leq k<n .
$$

We shall now present an amazingly simple formula for coefficients of the polynomial

$$
h(y):=\prod_{s=1}^{n-1}\left(y-i e^{\pi i s / n}\right)=\sum_{j=0}^{n-1} c_{j} y^{n-1-j}
$$


Proposition 2.1 let $\gamma_{k}:=\cot \left(\frac{k \pi}{2 n}\right)$. Then

$$
c_{0}=1, c_{j}=\prod_{k=1}^{j} \gamma_{k} \quad(1 \leq j \leq n-1)
$$

Proof .

Put $\xi_{k}=-i e^{\pi i k / n}, k=1, \ldots, n-1$. Then

$$
\begin{aligned}
c_{j} & =\text { the } \mathrm{j}-\text { th elementary symmetric function of } \xi_{1}, \ldots, \xi_{n-1} \\
& =e_{j}\left(\xi_{1}, \ldots, \xi_{n-1}\right)
\end{aligned}
$$

Let us first compute the power sums

$$
\begin{aligned}
p_{s}=\sum_{k=1}^{n-1} \xi_{k}^{s}=(-i)^{s} \sum_{k=1}^{n-1} e^{\pi i s k / n} & =(-i)^{s}\left(e^{\pi i s / n}-e^{\pi i s}\right) /\left(1-e^{\pi i s}\right) \\
& =\left\{\begin{array}{l}
(-1)^{\frac{s}{2}-1}, s \text { even } \\
(-1)^{\frac{s-1}{2}} \cot \left(\frac{s \pi}{2 n}\right)=(-1)^{\frac{s-1}{2}} \gamma_{s}, s \text { odd }
\end{array}\right.
\end{aligned}
$$

The proof will be by induction. For $j=1$ we have $c_{1}=\xi_{1}+\cdots+\xi_{n-1}=p_{1}=\gamma_{1}$. Suppose that the proposition is true for all $k<i$. Then by Newton formula for symmetric functions

$$
j e_{j}=\sum_{k=1}^{j}(-1)^{k-1} p_{k} e_{j-k}=\sum_{l=1}^{\lceil j / 2\rceil}\left(p_{2 l-1} e_{j-2 l+1}-p_{2 l} e_{j-2 l}\right)
$$


we obtain by writing $c_{j-2 l+1}=c_{j-2 l} \gamma_{j-2 l+1}$

$$
\begin{aligned}
j e_{j} & =\sum_{l=1}^{\lceil j / 2\rceil}\left((-1)^{l-1} \gamma_{2 l-1} \gamma_{j-2 l+1}-(-1)^{l-1}\right) c_{j-2 l} \\
& =\sum_{l=1}^{\lceil j / 2\rceil}(-1)^{l-1}\left(\gamma_{2 l-1} \gamma_{j-2 l+1}-1\right) c_{j-2 l} \\
& \stackrel{*}{=} \sum_{l=1}^{\lceil j / 2\rceil}(-1)^{l-1}\left(\gamma_{2 l-1}+\gamma_{j-2 l+1}\right) \gamma_{j} c_{j-2 l} \\
& =\sum_{l=1}^{\lceil j / 2\rceil}\left(p_{2 l-1} c_{j-2 l}-p_{2 l-2} \gamma_{j-2 l+1} c_{j-2 l}\right) \gamma_{j} \quad\left(\text { here } p_{0}:=-1\right) \\
& =\sum_{l=1}^{\lceil j / 2\rceil}\left(p_{2 l-1} c_{j-2 l}-p_{2 l-2} c_{j-2 l+1}\right) \gamma_{j} \\
& =\sum_{l=1}^{\lceil j / 2\rceil}\left(p_{2 l-1} c_{j-1-(2 l-1)}-p_{2 l-2} c_{j-1-(2 l-2)}\right) \gamma_{j} \\
& =\left(-p_{0} c_{j-1}+\sum_{l=1}^{\lceil(j-1) / 2\rceil}\left(p_{2 l-1} c_{j-1-(2 l-1)}-p_{2 l} c_{j-1-2 l}\right) \gamma_{j}\right. \\
& \stackrel{* *}{=}\left(c_{j-1}+(j-1) c_{j-1}\right) \gamma_{j} \\
& =j c_{j-1} \gamma_{j}=j c_{j}
\end{aligned}
$$

Here in $(*)$ we have used the cotangent addition formula $\cot (\alpha) \cot (\beta)-1=(\cot \alpha+$ $\cot \beta) \cot (\alpha+\beta)$ and in $(* *)$ Newton formula for $i-1$ which holds by induction hypothesis. The proposition is thus proved.

For our dihedral configurations we can state the stronger conjecture of Atiyah and Sutcliffe ([7], Conjecture 2.) as follows

$$
n^{\frac{n}{2}} \prod_{k=0}^{n-1} f_{k} \geq 2^{\left(\begin{array}{c}
n \\
2
\end{array}\right)} \prod_{i=0}^{n}\left(1+\lambda_{i}^{2}\right)^{n}
$$

where

$$
f_{k}=\sum_{s \geq 0}\left(\prod_{j=1}^{s} \lambda_{N-j n-k}^{n} \widetilde{E}_{k+s n}, \quad(0 \leq k<n)\right)
$$


From the factorization

$$
f(y)=h(y) \prod_{i=1}^{m}\left(y+\lambda_{i}\right)
$$

we can write

$$
\widetilde{E}_{k}=\sum_{i=0}^{n-1} c_{i} E_{k-i}
$$

in terms of elementary symmetric functions $E_{k}=e_{k}\left(\lambda_{1}, \ldots, \lambda_{m}\right)$ of our positive quantities $0<\lambda_{1}<\cdots<\lambda_{m}$ with coefficients $c_{i}$ given in Proposition 2.1 (note that $c_{0}=1 \leq c_{1} \leq \cdots \leq c_{\left\lfloor\frac{n-1}{2}\right\rfloor} \geq \cdots \geq c_{n-1}=1$ (unimodality) and $c_{i}=c_{n-1-i}$ (symmetry)).

Now we shall prove a generalization of the Đoković's conjecture which apparently strengthens (2.10).

Theorem 2.2 We have:

$$
\begin{aligned}
& \text { 1. } \prod_{k=0}^{n-1} f_{k} \geq \prod_{k=0}^{n-1} c_{k}\left(\sum_{l=0}^{m}\left(\prod_{j=0}^{l-1} \lambda_{m-j} E_{l}\right)\right)^{n} \\
& \text { 2. } \prod_{k=0}^{n-1} f_{k} \geq \prod_{k=0}^{n-1} c_{k} \prod_{i=1}^{m}\left(1+\lambda_{i}^{2}\right)^{n}
\end{aligned}
$$

\section{Proof .}

Let us write

$$
f_{k}=\sum_{l=0}^{m} \varphi_{k l} E_{l}
$$

Let us substitute $\widetilde{E}_{k+s n}=\sum_{i=0}^{n-1} c_{i} E_{k-i+s n}$ into (2.11). Then for fixed $k(0 \leq k<n-1)$ and given $l(0 \leq l \leq m)$ we seek $s \geq 0$ and $i, 0 \leq i<n$ such that $l=k-1+s n$, i.e. $l-k=s n-i, 0 \leq i<n$. We conclude that $s$ and $i$ are uniquely determined by a division algorithm (with nonpositive remainder):

$$
s_{k}:=\left\lceil\frac{l-k}{n}\right\rceil, \quad i_{k}=s_{k} n-l-k .
$$


Hence

$$
\varphi_{k l}=\prod_{j=1}^{s_{k}} \lambda_{N-j n-k}^{n} c_{i_{k}}
$$

with $s_{k}$ and $i_{k}$ just defined. It is easy to see that

$$
s_{k}=s_{0}\left(=\left\lceil\frac{l}{n}\right\rceil\right) \text { and } i_{k}=i_{0}+k \text { for } 0 \leq k \leq n-i_{0}-1
$$

and

$$
s_{k}=s_{0}-1 \text { and } i_{k}=i_{0}+k-n \text { for } n-i_{0} \leq k \leq n-1 .
$$

Lemma 2.3 For each $l, 0 \leq l \leq m$, we have

$$
\prod_{k=0}^{n-1} \varphi_{k l}=\prod_{j=0}^{l-1} \lambda_{m-j}^{n} \prod_{j=0}^{n-1} c_{j}
$$

Proof (of Lemma).

$$
\begin{aligned}
\prod_{k=0}^{n-1} \varphi_{k l} & =\prod_{k=0}^{n-i_{0}-1}\left(\prod_{j=1}^{s_{0}} \lambda_{N-j n-k}^{n} \prod_{k=i_{0}}^{n-1} c_{k}\right) \prod_{k=n-i_{0}}^{n-1} \prod_{j=1}^{s_{0}-1} \lambda_{N-j n-k}^{n} \prod_{k=0}^{i_{0}-1} c_{k} \\
& =\prod_{k=0}^{n-1} \prod_{j=1}^{s_{0}-1} \lambda_{N-j n-k}^{n-i_{0}-1} \prod_{k=0}^{n-1} \lambda_{N-s_{0} n-k}^{n} \prod_{k=0}^{n} c_{k}
\end{aligned}
$$

We put now $N=n+m$

$$
\begin{aligned}
& =\lambda_{m}^{n} \lambda_{m-1}^{n} \cdots \lambda_{m+n-s_{0} n-\left(n-i_{0}-1\right)}^{n} \prod_{k=0}^{n-1} c_{k} \\
& =\lambda_{m}^{n} \lambda_{m-1}^{n} \cdots \lambda_{m-l+1}^{n} \prod_{k=0}^{n-1} c_{k}
\end{aligned}
$$

Proof (of Theorem). 
We shall use the Hölder inequality

$$
\begin{aligned}
\prod_{k=0}^{n-1} f_{k}=\prod_{k=0}^{n-1}\left(\sum_{l=0}^{m} \varphi_{k l} E_{l}\right) & \geq\left(\sum_{l=0}^{m}\left(\prod_{k=1}^{n-1} \varphi_{k l} E_{l}\right)^{\frac{1}{n}}\right)^{n} \\
& =\left(\sum_{l=0}^{m} \prod_{j=0}^{l-1} \lambda_{m-j}\left(\prod_{j=0}^{n-1} c_{j}\right)^{\frac{1}{n}} E_{l}\right)^{n} \quad \text { (by lemma) } \\
& =\left(\prod_{j=0}^{n-1} c_{j}\right)\left(\sum_{l=0}^{m} \prod_{j=0}^{l-1} \lambda_{m-j} E_{l}\right)^{n}
\end{aligned}
$$

Thus 1. is proved. To obtain 2. we apply Đoković proof of Atiyah conjecture for type A configurations

$$
\sum_{l=0}^{m} \prod_{j=0}^{l-1} \lambda_{m-j} E_{l} \geq \prod_{i=1}^{m}\left(1+\lambda_{i}^{2}\right)
$$

(c.f. section 3.)

\section{Appendix}

After the first version of this paper was finished, in the meantime, we have discovered formulas for the partial derivatives, of the quantities $\Psi_{1 \ldots n}^{1 \ldots n} / \Psi_{1 \ldots \widehat{k} \ldots . . . n}^{1 \ldots .}$, with respect to variables $\xi_{r}$ (Note that in Theorem 1.8 we have given formulas w.r.t. variables $X_{r}$ !).

Lemma 3.1 For $2 \leq r \leq n$ the partial derivative w.r.t. $\xi_{r}$ of the quotient $\Psi_{1 \ldots n}^{1 \ldots n} / \Psi_{2 \ldots n}^{2 \ldots n}$ is given by

$$
\left(\Psi_{2 \ldots n}^{2 \ldots n}\right)^{2} \partial_{\xi_{r}}\left(\frac{\Psi_{1 \ldots n}^{1 \ldots n}}{\Psi_{2 \ldots n}^{2 \ldots n}}\right)=\sum_{i \geq j} s_{i j}^{\prime} X_{1}\left(X_{2} \cdots X_{j}\right)^{2} X_{j+1} \cdots X_{i+1}\left(X_{j+1}-X_{i+2}\right)
$$

where $s_{i j}^{\prime}$ is the conjugated Schur function $s_{i j}=s_{i j}\left(\xi_{2}, \ldots, \xi_{r-1}, \xi_{r+1}, \ldots, \xi_{n}\right)$ corresponding to a two-rowed partition $\lambda=(i \geq j)$.

In particular for $X_{1} \geq \cdots \geq X_{n}>0$ the function $\Psi_{1 \ldots n}^{1 \ldots n} / \Psi_{2 \ldots n}^{2 \ldots n}$ is monotonically increasing w.r.t. the variable $\xi_{r}$ (for $r=1$, too). 
Proof .

By using the formula $\Psi_{1 \ldots n}^{1 \ldots n}=\Psi_{1 \ldots \widehat{r} \ldots n}^{1 \ldots n-1}+X_{1} \xi_{r} \Psi_{1 \ldots \widehat{r} \ldots n}^{2 \ldots n}$ we get

$$
\begin{aligned}
& \partial_{\xi_{r}}\left(\Psi_{1 \ldots n}^{1 \ldots n}\right) \Psi_{2 \ldots n}^{2 \ldots n}-\Psi_{1 \ldots n}^{1 \ldots n} \partial_{\xi_{r}}\left(\Psi_{2 \ldots n}^{2 \ldots n}\right)=
\end{aligned}
$$

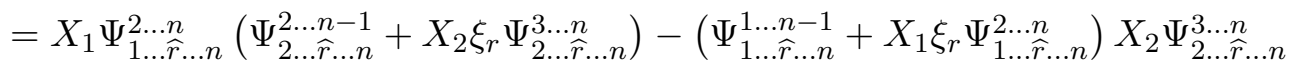

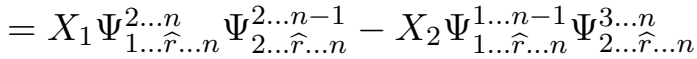

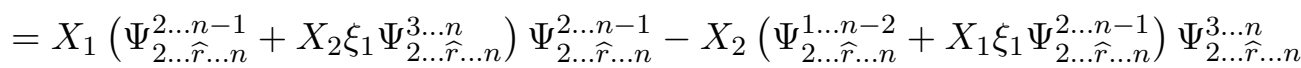

$$
\begin{aligned}
& =X_{1}\left(\Psi_{2 \ldots \widehat{r} \ldots n}^{2 \ldots . . n-2}\right)^{2}-X_{2} \Psi_{2 \ldots . . . . n}^{1 \ldots n-2} \Psi_{2 \ldots . . . . n}^{3 \ldots n}
\end{aligned}
$$

With $e_{i}=e_{i}^{(1 r)}=e_{i}\left(\xi_{2}, \ldots, \xi_{r-1}, \xi_{r+1}, \ldots, \xi_{n}\right)$ denoting the $i$-th elementary symmetric function of the truncated alphabet $A^{(1 r)}=\left\{\xi_{2}, \ldots, \xi_{r-1}, \xi_{r+1}, \ldots, \xi_{n}\right\}$ we have further

$$
\begin{aligned}
& =X_{1}\left(\sum_{i, j} e_{i} e_{j} X_{2 \ldots i+1} X_{2 \ldots j+1}\right)-X_{2}\left(\sum_{i, j} e_{i} e_{j} X_{1 \ldots i} X_{3 \ldots j+2}\right) \\
& =\sum_{i, j} e_{i} e_{j} X_{1 . . i+1} X_{2 . . j+1}-\sum_{i, j} e_{i} e_{j} X_{1 . . i} X_{2 . . j+2} \\
& =\sum_{i, j}\left|\begin{array}{cc|l}
e_{i} & e_{i+1} \\
e_{j-1} & e_{j}
\end{array}\right| X_{1 . . i+1} X_{2 . . j+1} \\
& =\sum_{i \geq j}\left|\begin{array}{cc|}
e_{i} & e_{i+1} \\
e_{j-1} & e_{j}
\end{array}\right| X_{1}\left(X_{2 . . j}\right)^{2} X_{j+1} \cdots X_{i+1}\left(X_{j+1}-X_{i+2}\right)
\end{aligned}
$$

Now by Jacobi-Trudy formula we can write $\left|\begin{array}{cc}e_{i} & e_{i+1} \\ e_{j-1} & e_{j}\end{array}\right|$ as the conjugated Schur function $s_{i j}^{\prime}={s^{\prime}}_{i j}^{(1 r)}$ corresponding to a partition $(i \geq j)$.

Corollary 3.2 ( $\xi_{n}-$ monotonicity)

We have the following inequality:

$$
\frac{\Psi_{1 \ldots n}^{1 \ldots n}}{\Psi_{2 \ldots n}^{2 \ldots n}} \geq \frac{\Psi_{1 \ldots n-1}^{1 \ldots n-1}}{\Psi_{2 \ldots n-1}^{2 \ldots n n-1}}
$$

\section{Proof .}

By Lemma 3.1 by letting $\xi_{n} \downarrow 0$ we get

$$
\Psi_{1 \ldots n}^{1 \ldots n} / \Psi_{2 \ldots n}^{2 \ldots n} \geq \Psi_{1 \ldots n}^{1 \ldots n} /\left.\Psi_{2 \ldots n}^{2 \ldots n}\right|_{\xi_{n}=0}=\Psi_{1 \ldots n-1}^{1 \ldots n-1} / \Psi_{2 \ldots n-1}^{2 \ldots n-1}
$$


By using this Corollary we state a strengthening of our Conjecture 1.5:

\section{Conjecture 3.3}

$$
\left(\Psi_{1 \ldots n}^{1 \ldots n}\right)^{n-2} \geq \Psi_{2 \ldots n-2}^{2 \ldots n-1} \prod_{k=2}^{n-1} \Psi_{1 \ldots \widehat{k} \ldots n}^{1 \ldots . . .} \ldots n
$$

We also have formulas for partial derivative of the quotient $\Psi_{1 \ldots n}^{1 \ldots n} / \Psi_{1 \ldots \widehat{k} \ldots n}^{1 \ldots . . .}$ w.r.t. variable $\xi_{r}, 2 \leq r \leq n$, which are more complicated than for $k=1$ (given in Lemma 3.1). Without loss of generality we take $r=n$ and proceed as follows:

$$
\begin{aligned}
& \partial_{\xi_{n}}\left(\Psi_{1 \ldots n}^{1 \ldots n}\right) \Psi_{1 \ldots \widehat{k} \ldots n}^{1 \ldots . \widehat{k} \ldots n}-\Psi_{1 \ldots n}^{1 \ldots n} \partial_{\xi_{n}}\left(\Psi_{1 \ldots \widehat{k} \ldots n}^{1 \ldots \widehat{k} \ldots n}\right)= \\
& =X_{1} \Psi_{1 \ldots n-1}^{2 \ldots . . n} \Psi_{1 \ldots \widehat{k} \ldots n}^{1 \ldots \widehat{k} \ldots n}-X_{1} \Psi_{1 \ldots n}^{1 \ldots n} \Psi_{1 \ldots \widehat{k} \ldots n-1}^{2 \ldots \widehat{k} \ldots n} \\
& =X_{1} \Psi_{1 \ldots n-1}^{2 \ldots . n}\left(\Psi_{1 \ldots \widehat{k} \ldots . . .}^{1 \ldots \widehat{k}}+X_{1} \xi_{n} \Psi_{1 \ldots \widehat{k} \ldots n-1}^{2 \ldots \widehat{k} \ldots n}\right)-X_{1}\left(\Psi_{1 \ldots n-1}^{1 \ldots . . n-1}+X_{1} \xi_{n} \Psi_{1 \ldots n-1}^{2 \ldots n}\right) \Psi_{1 \ldots \widehat{k} \ldots n-1}^{2 \ldots . . \widehat{k} \ldots n} \\
& =X_{1}\left(\Psi_{1 \ldots n-1}^{2 \ldots n} \Psi_{1 \ldots \widehat{k} \ldots n-1}^{1 \ldots \widehat{k} \ldots n-1}-\Psi_{1 \ldots n-1}^{1 \ldots n-1} \Psi_{1 \ldots \widehat{k} \ldots n-1}^{2 \ldots . . . . n}\right) \\
& =X_{1}\left[\left(\Psi_{1 \ldots \widehat{k} \ldots n-1}^{2 \ldots . n-1}+X_{2} \xi_{k} \Psi_{1 \ldots \widehat{k} \ldots n-1}^{3 \ldots . n}\right) \Psi_{1 \ldots \widehat{k} \ldots . . . n-1}^{1 \ldots \widehat{k} \ldots n-1}\right. \\
& \left.-\left(\Psi_{1 \ldots \widehat{k} \ldots n-1}^{1 \ldots n-2}+X_{1} \xi_{k} \Psi_{1 \ldots \widehat{k} \ldots n-1}^{2 \ldots . n-1}\right) \Psi_{1 \ldots \widehat{k} \ldots n-1}^{2 \ldots . \widehat{k} \ldots n}\right] \\
& =X_{1}\left[\Psi_{1 \ldots \widehat{k} \ldots n-1}^{2 \ldots . . n-1} \Psi_{1 \ldots \widehat{k} \ldots n-1}^{1 \ldots \widehat{k} \ldots n-1}-\Psi_{1 \ldots \widehat{k} \ldots n-1}^{1 \ldots . . . n-2} \Psi_{1 \ldots \widehat{k} \ldots . . n-1}^{2 \ldots \widehat{k} \ldots n}+\right. \\
& \left.+\xi_{k}\left(X_{2} \Psi_{1 \ldots \widehat{k} \ldots n-1}^{3 \ldots n} \Psi_{1 \ldots \widehat{k} \ldots n-1}^{1 \ldots \widehat{k} \ldots n-1}-X_{1} \Psi_{1 \ldots \widehat{k} \ldots n-1}^{2 \ldots n-1} \Psi_{1 \ldots \widehat{k} \ldots n-1}^{2 \ldots . \widehat{k} \ldots n}\right)\right] \\
& =X_{1}\left[I_{1}-\xi_{k} I_{2}\right]
\end{aligned}
$$


Now we first compute

$$
\begin{aligned}
& I_{1}=\Psi_{1 \ldots \widehat{k} \ldots n-1}^{2 \ldots . n-1} \Psi_{1 \ldots . . . . . n-1}^{1 \ldots . \widehat{k} \ldots n-1}-\Psi_{1 \ldots \widehat{k} \ldots n-1}^{1 \ldots n-2} \Psi_{1 \ldots \widehat{k} \ldots . . n-1}^{2 \ldots \widehat{k} \ldots n}= \\
& \left(\sum_{i=0}^{k-2} e_{i} X_{2 . . i+1}+\sum_{i=k-1}^{n-2} e_{i} X_{2 . . i+1}\right)\left(\sum_{j=0}^{k-1} e_{j} X_{1 . . j}+\sum_{j=k}^{n-2} e_{j} X_{1 . . \widehat{k} . . j+1}\right)- \\
& -\left(\sum_{j=0}^{k-1} e_{j} X_{1 . . j}+\sum_{j=k}^{n-2} e_{j} X_{1 . . j}\right)\left(\sum_{i=0}^{k-2} e_{i} X_{2 . . i+1}+\sum_{i=k-1}^{n-2} e_{i} X_{2 . \widehat{k} . . i+2}\right)= \\
& =\sum_{i=k-1}^{n-2} \sum_{j=0}^{k-1} e_{i} e_{j}\left(X_{2 . . i+1} X_{1 . . j}-X_{2 . . \widehat{k} . . i+1} X_{1 . . j}\right)+ \\
& +\sum_{j=k}^{n-2} \sum_{i=0}^{k-2} e_{j} e_{i}\left(X_{1 . . \widehat{k} . . j+1} X_{2 . . i+1}-X_{1 . . j} X_{2 . . i+1}\right)+ \\
& +\sum_{i=k-1}^{n-2} \sum_{j=k}^{n-2} e_{i} e_{j}\left(X_{2 . . i+1} X_{\left.1 . . \widehat{k}_{. . j+1}-X_{1 . . j} X_{2 . . \widehat{k} . . i+2}\right)}\right.
\end{aligned}
$$

By replacing, in the middle sum, $j$ with $i+1$ and $i$ with $j-1$, and observing that then $X_{1 . . \widehat{k} . . i+2} X_{2 . . j}-X_{1 . . i+1} X_{2 . . j}=-\left(X_{2 . . i+1} X_{1 . . j}-X_{2 . . \widehat{k} . . i+2} X_{1 . . j}\right)$ the contribution of the first two sums is

$$
\sum_{i=k-1}^{n-2} \sum_{j=0}^{k-1}\left|\begin{array}{cc}
e_{i} & e_{i+1} \\
e_{j-1} & e_{j}
\end{array}\right| X_{2 . \widehat{k} . . i+1}\left(X_{k}-X_{i+2}\right) X_{1 . . j}
$$

The third sum can similarly be transformed to the following form:

$$
\sum_{k \leq j \leq i \leq n-2}\left|\begin{array}{cc}
e_{i} & e_{i+1} \\
e_{j-1} & e_{j}
\end{array}\right| X_{2 . . k_{. . i+1}}\left(X_{j+1}-X_{i+2}\right) X_{1 . . j}
$$

Hence

$$
I_{1}=\sum_{0 \leq j, \max \{j, k-1\} \leq i \leq n-2} s_{i j}^{\prime} X_{2 . . \widehat{k}_{. . i+1}}\left(X_{\max \{j+1, k\}}-X_{i+2}\right) X_{1 . . j} \quad(\geq 0)
$$

By a similar manipulation we can obtain the expression for the quantity

$$
\begin{aligned}
& I_{2}=X_{1} \Psi_{1 \ldots \widehat{k} \ldots n-1}^{2 \ldots . n-1} \Psi_{1 \ldots \widehat{k} \ldots n-1}^{2 \ldots \widehat{k} \ldots n}-X_{2} \Psi_{1 \ldots \widehat{k} \ldots n-1}^{3 \ldots . . .} \Psi_{1 \ldots \widehat{k} \ldots n-1}^{1 \ldots \widehat{k} \ldots n-1}= \\
& =X_{1}-X_{2}+\sum_{i=1}^{n-1} \sum_{j \leq \min \{k-1, i\}} s_{i j}^{\prime} X_{2 . . \widehat{k} . . i+2} X_{1 . . j}\left(X_{j+1}-X_{k}\right) \geq 0
\end{aligned}
$$


where $s_{i j}^{\prime}$ is conjugated Schur function $s_{i j}^{\prime}={s^{\prime}}_{i j}^{(k n)}$. We see that

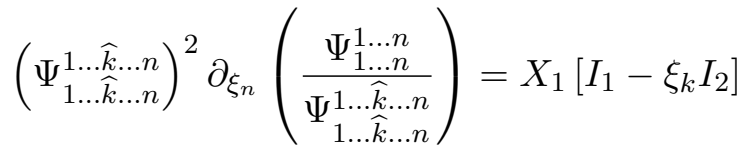

has both positive and negative terms. And we have not been able to apply it so far.

Now we illustrate use of $\xi$-monotonicity (in addition to $X$-monotonicity) for proving once more the case $n=4$ of our Conjecture 1.5:

$$
\begin{aligned}
& \frac{\left(\Psi_{1234}^{1234}\right)^{3}}{\Psi_{234}^{234} \Psi_{134}^{134} \Psi_{124}^{124} \Psi_{123}^{123}}=\frac{\Psi_{1234}^{1234}}{\Psi_{234}^{234} \Psi_{123}^{123}} \frac{\Psi_{1234}^{1234}}{\Psi_{134}^{134}} \frac{\Psi_{1234}^{1234}}{\Psi_{124}^{124}} \geq\left(\text { by } \xi_{4} \text {-monotonicity }\right) \\
& \geq \frac{1}{\Psi_{23}^{23}} \frac{\Psi_{1234}^{1234}}{\Psi_{134}^{134}} \frac{\Psi_{1234}^{1234}}{\Psi_{124}^{124}} \geq\left(\text { by } X_{1} \text {-monotonicity twice and } X_{4} \text {-monotonicity }\right) \\
& \geq \frac{1}{\Psi_{23}^{23}} \frac{\Psi_{1243}^{2234}}{\Psi_{143}^{234}} \frac{\Psi_{1234}^{2233}}{\Psi_{124}^{223}} \geq \text { (by } \xi_{3} \text {-monotonicity) } \\
& \geq \frac{1}{\Psi_{23}^{23}} \frac{\Psi_{124}^{223}}{\Psi_{14}^{23}} \frac{\Psi_{1234}^{2233}}{\Psi_{124}^{223}}=\frac{\Psi_{1234}^{2233}}{\Psi_{23}^{23} \Psi_{14}^{23}} \geq 1
\end{aligned}
$$

Similarly the cases $n=5,6,7$ of Conjecture 1.5 would be, by using $\xi$-monotonicity and $X$-monotonicity, consequences of the following inequalities

$$
\widetilde{Q}_{n} \geq 1
$$

where

$$
\begin{aligned}
& \widetilde{Q}_{5}=\Psi_{12345}^{22344} \Psi_{12345}^{22344} / \Psi_{234}^{234} \Psi_{135}^{234} \Psi_{1245}^{2244} \\
& \widetilde{Q}_{6}=\Psi_{123456}^{223445} \Psi_{123456}^{233455} / \Psi_{2345}^{2345} \Psi_{1346}^{2345} \Psi_{1256}^{2345} \\
& \widetilde{Q}_{7}=\Psi_{1234567}^{2234556} \Psi_{1234567}^{2334566} \Psi_{1234567}^{2344566} / \Psi_{23456}^{23456} \Psi_{13457}^{23456} \Psi_{12467}^{23456} \Psi_{123567}^{234566}
\end{aligned}
$$

\subsection{Computer verification of the Conjecture 1.5 (and hence of the Atiyah-Sutcliffe conjecture C3) for almost collinear $9+1$ con- figuration.}

Let us now explain our computer verification of the inequality $\widetilde{Q}_{9} \geq 1$ where

$$
\widetilde{Q}_{9}=\frac{\Psi_{123456789}^{223456778} \Psi_{123456789}^{233456788} \Psi_{123456789}^{22345678} \Psi_{123456789}^{234456788}}{\Psi_{2345678}^{2345678} \Psi_{1345679}^{2345678} \Psi_{1245689}^{234567} \Psi_{1235789}^{2345678} \Psi_{12346789}^{2234678}}
$$


which refines the case $n=9$ of the Conjecture 1.5 . We have observed first that $\widetilde{Q}_{9}$ is symmetric in partial alphabets

$$
A_{1}=\left\{\xi_{1}, \xi_{2}, \xi_{8}, \xi_{9}\right\}, A_{2}=\left\{\xi_{3}, \xi_{4}, \xi_{6}, \xi_{7}\right\}, A_{3}=\left\{\xi_{5}\right\}
$$

then by introducing the elementary symmetric functions $\left\{e_{1}, e_{2}, e_{3}, e_{4}\right\}$ of $A_{1}$ and $\left\{f_{1}, f_{2}, f_{3}, f_{4}\right\}$ of $A_{2}$ we first computed the products

$$
\Psi_{2345678}^{2345678} \Psi_{1345679}^{2345678} \text { and } \Psi_{1245689}^{2345678} \Psi_{1235789}^{2345678}
$$

in terms of $\left\{e_{1}, e_{2}, e_{3}, e_{4}, f_{1}, f_{2}, f_{3}, f_{4}, \xi_{5}\right\}$. Then by successive application of Stembridge's Maple SF package we expressed the difference $\Delta:=\operatorname{numer}\left(\widetilde{Q}_{9}\right)-\operatorname{denom}\left(\widetilde{Q}_{9}\right)$ in terms of the Schur functions of both alphabets $A_{1}$ and $A_{2}$. Then we factored each coefficient in such a multi-Schur expansion and into non-monomial factors we substituted $X_{2}=X_{3}+h_{2}, X_{3}=X_{4}+h_{3}, \ldots, X_{7}=X_{8}+h_{7}$. Then the computation showed that the coefficients of all monomials in $X_{8}, h_{2}, \ldots, h_{7}$ were nonnegative. The factoring out the trivial monomial factors in $X_{2}, \ldots, X_{8}$ (which are trivially nonnegative) was crucial because otherwise the expansion of multi-Schur function coefficients in terms of increments $h_{2}, \ldots, h_{7}$ may not be feasible.

\section{References}

[1] M. Atiyah. The geometry of classical particles. Surveys in Differential Geometry (International Press) 7 (2001).

[2] M. Atiyah. Configurations of points. Phil. Trans. R. Soc. Lond. A 359 (2001), $1375-1387$.

[3] M. Atiyah and P. Sutcliffe. Polyhedra in Physics, Chemistry and Geometry, Milan Journal of Mathematics, Vol 71, Number 1/September 2003, 33-58

[4] M. Atiyah and P. Sutcliffe. The geometry of point particles. Royal Society of London Proceedings Series A, vol. 458, Issue 2021., 1089.-1116.

[5] M. Eastwood and P. Norbury, A proof of Atiyah's conjecture on configurations of four points in Euclidean three-space. Geometry \& Topology 5 (2001), 885-893.

[6] D.Ž. Đoković, Proof of Atiyah's conjecture for two special types of configurations, arXiv:math.GT/0205221 v4, 11 June 2002. Electron. J. Linear Algebra 9 (2002), $132-137$. 
[7] D.Ž. Đoković, D. Ž., Verification of Atiyah's conjecture for some nonplanar configurations with dihedral symmetry.In Publ. Inst. Math., Nouv. Ser. 72(86), (2002) 23-28.

[8] I. G. Macdonald Symmetric functions and Hall polynomials $2^{\text {nd }}$ edition, Oxford University Press, 1995.

[9] Joseph Malkoun, On the Atiyah problem on Hiperbolic Configurations of Four Points, arXive:150201364, 2015.; Geometrie Dedicata 2016., Vol 180, pp 287-292

[10] Mazen Bou Khusan, Michael Johnson On the conjecture regarding the fourpoint Atiyah determinant, SIGMA 10 (2014.), 070, 9 pages

[11] D. Svrtan, I. Urbiha Atiyah-Sutcliffe Conjectures for Almost Collinear Configurations and Some New Conjectures for Symmetric Functions, arXiv:math/0406386 (23 pages)

[12] D. Svrtan, I. Urbiha Verification and Strengthening of the Atiyah-Sutcliffe Conjectures for several types of Configurations, arXiv:math/0609174 (49 pages)

[13] D. Svrtan, Intrinsic Formula for Five Point (in euclidean plane) Atiyah Determinant, https://www.bib.irb.hr/553790 (2010)

[14] D. Svrtan, A proof of All three Euclidean Four Point Atiyah-Sutcliffe Conjectures, https://emis.de/journals/SLC/wpapers/s73vortrag/svrtan.pdf

[15] D. Svrtan, A proof of All three Atiyah-Sutcliffe 4-point Conjectures, International Congress of Mathematicians, Seoul 2014, Abstracts Short Communication Poster Sessions, 136-137, S. Korea

[16] D. Svrtan, A progress on Atiyah-Sutcliffe geometric conjectures, Math-ChemComp, 2007., Dubrovnik, June 11.-16. 
